\title{
The Toxicity of 35 Trace Elements in Coal to Freshwater Biota: A Data Base with Automated Retrieval Capabilities
}

\author{
R. M. Cushman \\ S. G. Hildebrand \\ R. H. Strand \\ R. M. Anderson
}

Prom

Publication No. 1048

\section{OAK RIDGE NATIONAL LABORATORY}




\section{DISCLAIMER}

This report was prepared as an account of work sponsored by an agency of the United States Government. Neither the United States Government nor any agency Thereof, nor any of their employees, makes any warranty, express or implied, or assumes any legal liability or responsibility for the accuracy, completeness, or usefulness of any information, apparatus, product, or process disclosed, or represents that its use would not infringe privately owned rights. Reference herein to any specific commercial product, process, or service by trade name, trademark, manufacturer, or otherwise does not necessarily constitute or imply its endorsement, recommendation, or favoring by the United States Government or any agency thereof. The views and opinions of authors expressed herein do not necessarily state or reflect those of the United States Government or any agency thereof. 


\section{DISCLAIMER}

Portions of this document may be illegible in electronic image products. Images are produced from the best available original document. 


\section{Printed in the United States of America. Available from National Technical Information Service \\ U.S. Department of Commerce \\ 5285 Port Royal Road, Springfield, Virginia 22161 \\ Price. Prinled Cupy $\$ 4.50$, Micrúficlie $\$ 3.00$}

This report was prepared as an account of work sponsored by the United States Government. Neither the United States nor the Energy Research and Development Administration/United States Nuclear Regulatory Commission, nor any of their employees, nor any of their contractors, subcontractors, or their employees, makes any warranty, express or implied, or assumes any legal liability or responsibility for the accuracy, completeness or usefulness of any information, apparatus, product or process disclosed, or represents that its use would not infringe privately owned rights. 
THE TOXICITY OF 35 TRACE ELEMENTS IN COAL TO FRESHWATER BIOTA:

A DATA BASE WITH AUTOMATED RETRIEVAL CAPABILITIES

R. M. Cushman, S. G. Hildebrand,

R. H. Strand, and R. M. Anderson

ENVIRONMENTAL SCIENCES DIVISION

Publication No. 1048

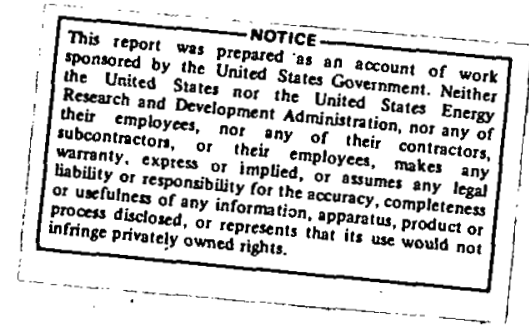

Date Published - June 1977

OAK RIDGE NATIONAL LABORATORY

Oak Ridge, Tennessee 37830 operated by

UNION CARBIDE CORPORATION

for the

FNERGY RESEARCH AND DEVELOPMENT ADMINISTRATION 
THIS PAGE

WAS INTENTIONALLY

LEFT BLANK 


\section{ACKNOWLEDGEMENTS}

The authors would like to acknowledge R. J. 0lson and L. W. Barnthouse, of the Environmental Sciences Division, ORNL, who critically reviewed this manuscript.

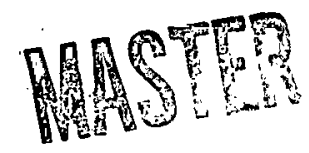

DISTRIBUTION OF THIS DOCUMENT IS UNLIMITEO

$e \beta$ 
THIS PAGE WAS INTENTIONALLY LEFT BLANK 
CUSHMAN, R. M., S. G. HILDEBRAND, R. H. STRAND, and R. M. ANDERSON. 1977. The toxicity of 35 trace elements in coal to freshwater biota: a data base with automated retrieval capabilities. ORNL/TM-5793. Oak Ridge National Laboratory, Oak Ridge, Tennessee. $46 \mathrm{pp}$.

Data are tabulated on the toxicity to freshwater biota of 35 trace elements with the potential for release to the environment from coal conversion effluents. The entire data base is presented on a microfiche appended to this document, in the interest of portability and accessibility. The data were gathered from a variety of research papers, compendia, and reviews. Details of water chemistry and test conditions are presented when available from the documents consulted. The data base may be used by referring directly to the tabulated data as they appear on the microfiche, or, with appropriate computer facilities, by manipulation (sorting, subsetting, or merging) of the data to meet the particular needs of the investigator. The data may be used as they appear in the data base, or the data base may be used to index the cited original papers. 


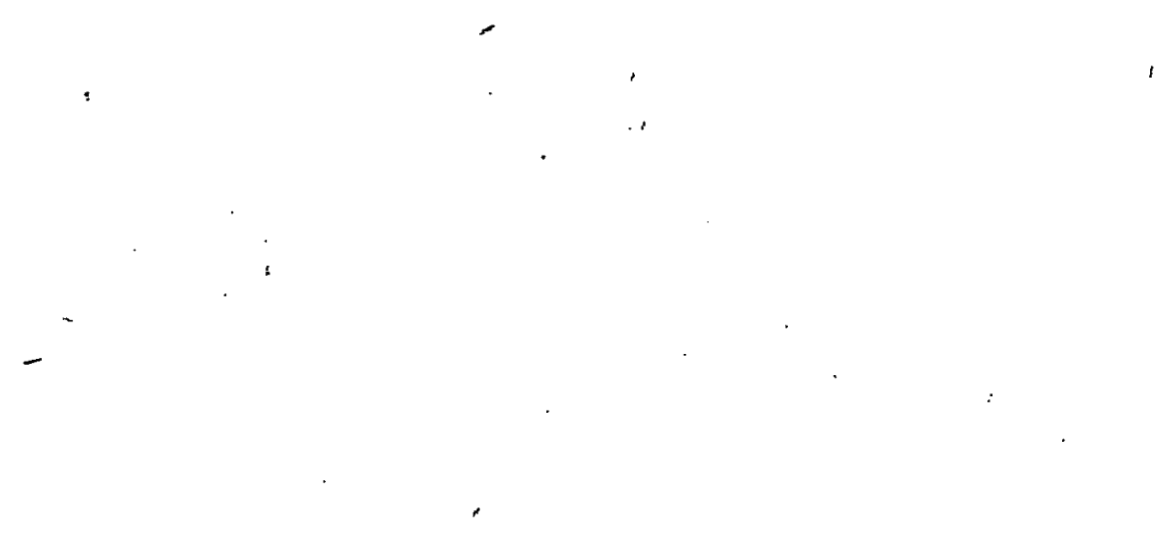

THIS PAGE

WAS INTENTIONALLY

LEFT BLANK 


\section{TABLE OF CONTENTS}

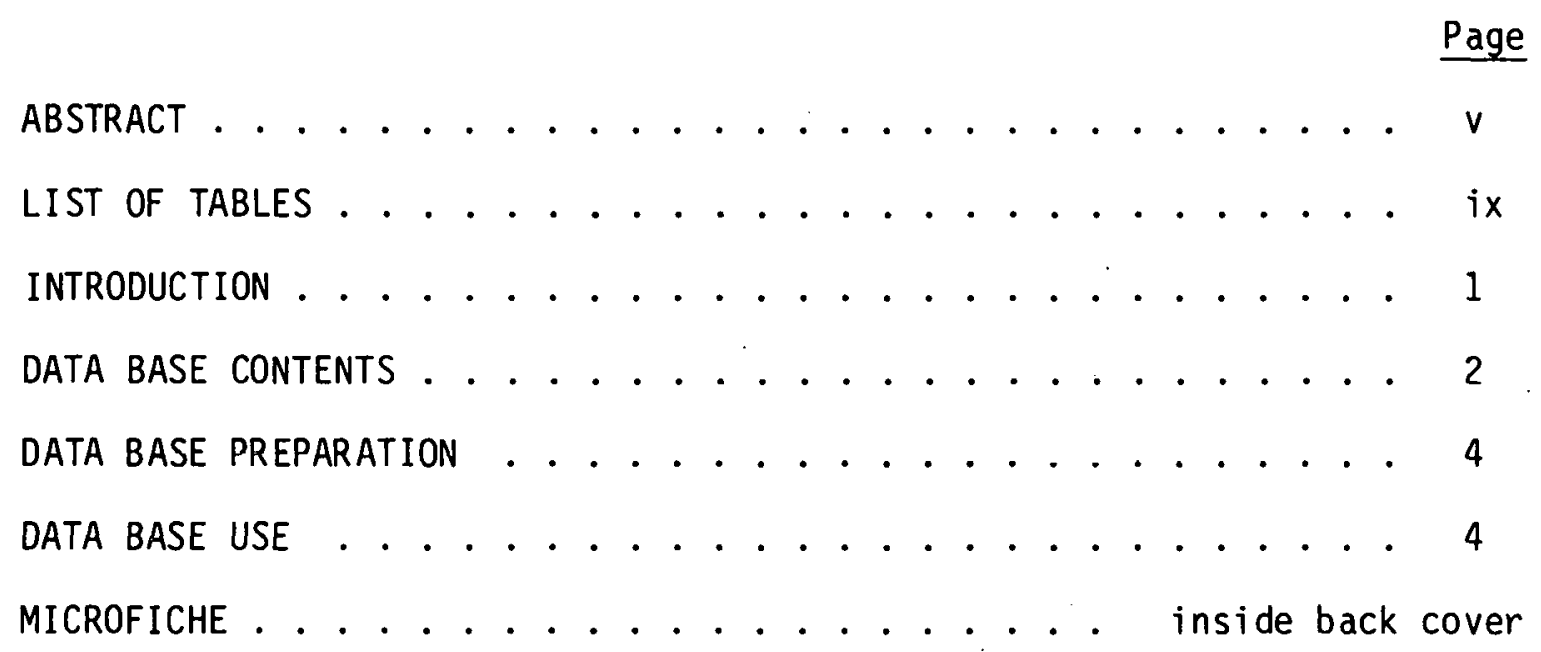


THIS PAGE

WAS INTENTIONALLY

LEFT BLANK 


\section{LIST OF TABLES}

$\underline{\text { Table }}$

$\underline{\text { Page }}$

1 Elements included in the toxicity data base . . . . . . 6

2 Variables and variable definitions used in the data base . 7

3 Frequency of occurrence of water chemistry and test conditions variables for each element........... 8

4 Sample page from data base . . . . . . . . . . . . 10

5 Scientific and common names of organisms in toxicity table . 11

6 Chemical form symbols as they appear on the microfiche and the corresponding chemical formulas . . . . . . . . . 15

7 List of references used to document the data presented in the toxicity data base .............. 17

8 Program and output listing to subset data base for only those records with an organism name within crustaceans

A. Program listing .. . . . . . . . . . . . . 40

B. Portion of output from previous program . . . . . . . 41 


\section{INTROOUCTION}

At least 30 trace elements have been detected in measurable quantities in potential aqueous effluents from coal conversion processes. 1 For elements such as chromium, copper, and zinc, there exists an extensive literature describing the toxic effects on aquatic biota. However, for the majority of elements, the data are less abundant, less available, and scattered throughout a diverse literature of compendia, review papers, and papers describing original research. It is, therefore, difficult for an individual to readily assemble the available toxicity data in order to assess, for example, the potential toxicity of a given concentration of a particular element. Reliance upon one or more of the standard compendia restricts data extraction to that information contained in the compendia. Furthermore, details of the experimental conditions (water chemistry, type of bioassay employed, etc.) may or may not be provided. Maintaining a current knowledge of the burgeoning toxicity literature is also unrealistic for most researchers, if on $7 y$ because of the time consumed.

This effort represents the gathering of trace element toxicity data from a wide variety of sources into a single computerized data base. Data were gathered both from original research papers and from summary and review documents. The 35 elements listed in Table 1 were used in the literature search for available toxicity information, as they had potential for release to the environment from coal conversion process effluents and they could be toxic to freshwater biota. The concentration of the element and details of the bioassay or field conditions were extracted. Such conditions as water chemistry, life stage, and observed toxic effect were extracted in addition to other useful information. In our data extractions from the literature, we consistently sought values for the set of variables in the computerized data base. Table 2 lists and defines the variables used in the data base. Certain variables unique to a research paper were not included in the data base due to space requirements.

Details of the bioassay or field situation used in the paper were extracted when available since water chemistry and test conditions would be necessary for interpretation and application of the data. However, details such as these were frequently not included in summary documents. Table 3 shows the percent frequency of occurrence over all observations for which a value for each of the variables described in Table 2 was

${ }^{1}$ S. G. Hildebrand, R. M. Cushman, and J. A. Carter. The potential toxicity and bioaccumulation in aquatic systems of trace elements present in aqueous coal conversion effluents. Proc. of 10th Ann. Conf. on Trace Substances in Environmental Health, Columbia, Mo, June 8-10, 1976, D. D. Hemphill, ed., in press. 
reported. These percentages are listed by element and range from 0 to $100 \%$ depending on the element and the variable. Where additional descriptions of the research are desired, we refer the user to the original references.

This report describes the data base we have formulated and computer ized and shows a typical use and application of the data base for determining the effects of certain trace elements on biota. Since the data base is in the preliminary stages, we expect that (a) the user will find documents from which we have not extracted data, (b) this data base will be expanded as funding permits, and (c) applications will be more extensive and useful as more data are added. We present the data base in an abbreviated form below to show the user what kinds of data were extracted. The complete data base, together with concise descriptions of the variables, the frequency table (Table 3), and references are supplied on microfiche in the pocket insert (inside back cover). We believe that this form of the entire data base is more portable and accessible than a volumnious printout of the entire data base. With proper attention paid to this report, the reader should be able to use just the microfiche for subsequent access to the data base.

\section{DATA BASE CONTENTS}

What follows is a description of what is contained in the data base as presented in its entirety on the microfiche contained in the pocket insert. We will describe the data base as an item contained within the microfiche. The microfiche includes:

Item 1. definitions of the variable names used in the data tables;

Item 2. a table of frequency of occurrence of data for each of the variables in the data base, by element;

Item 3. the entire data base in tabular form, with an observation number assigned to each record;

Item 4. the observations sorted alphabetically by organism, with the element, its chemlcal form, and a cross-reference to the observation number in the entire data base (Item 3); and

Item 5. the list of references from which data were extracted and which are listed as variables in the entire data base (Item 3) to provide a cross-reference between the data base and the references.

Items $1,2,4$, and 5 are descriptions, definitions, or rearrangements of the entire data base (Item 3 ). They are necessary for completing the microfiche as an exportable and usable data base. We have presented a sample of the entire data base in Table 4. 
Data are presented both for lethal and sublethal effects. Data in the form LCX signify the lethal concentration for $X$ percent of the organisms tested in the stated time period, if a time is specified, as in a 96-hr LC50. Endpoints reported as ILL (incipient lethal level) indicate threshold effects or concentrations causing mortality with theoretically infinite exposure. Sublethal effects include impairment of reproduction, growth, behavior, etc. For a rigorous discussion of bioassays and toxicity terminology the reader is referred to the series "Measurement of pollutant toxicity to fish," by J.B. Sprague, published in three parts:

(1969) I. Bioassay methods for acute toxicity. Water Research 3:793-821.

(1970) II. Utilizing and applying bioassay results. Water Research $4: 3-32$.

(1971) III. Sublethal effects and "safe" concentrations. Water Research 5:245-266.

In addition to the element and its concentration (mg/1), test organism (species and life stage), and toxicity end point, we have tabulated water chemistry parameters and other test conditions, including:

(1) type and source of dilution water

(2) whether the test was static or continuous flow

(3) temperature $\left({ }^{0} \mathrm{C}\right)$

(4) hardness $(\mathrm{mg} / 1)$

(5) $\mathrm{pH}$

(6) calcium concentration (mg/1)

(7) magnesium concentration $(\mathrm{mg} / 1)$

(8) dissolved oxygen (DO) ( $\mathrm{mg} / 1$ )

(9) total dissolved solids (TDS) (mg/1)

(10) alkalinity (mg/1)

(11) misce 11 aneous comments

(12) form of element or chemical compound used

For some of the variables (concentration, temperature, hardness, $\mathrm{pH}$, dissolved oxygen, total dissolved solids, and alkalinity), a range of values was reported, and minimum and maximum values of the variable(s) are tabulated in the data base. However, where only a single value appears (as either a minimum or a maximum) a range was not. reported, and the listed value does not necessarily represent a minimum or a maximum. Where a value for a variable was not present in the report, a period is recorded in the data tables.

For each observation, two reference citations are given, one representing the source(s) from which we directly obtained the data (variables OUR_REF1, OUR_REF2, and OUR_REF3), and the other representing 
the source(s) in which the data first were reported (variables REFIORIG, REF20RIG, and REF30RIG). The values for these variables, tabulated in the data base (Item 3 ), correspond to the numbers preceding each reference in the list of references (Item 5). Thus, the cross-reference between the two items in the data base. Table 5 lists the scientific (and common) names used in the data base, grouped into fish, protozoa, algae, other plants, crustacea, insecta, mollusca, and other animals. Table 6 relates the printout of the chemical form symbols as they appear on the microfiche to the appropriate chemical formulas. Table 7 is the list of references, which also appears in the microfiche output.

\section{DATA BASE PREPARATION}

Following the selection of a reference and the itemization of the test conditions used in the bioassay, the data were encoded in a format with a varying amount of space allowed for each variable. Once computerized, each observation consisted of multiple 80-column card images. An initial file was maintained a MODCOMP II/25 computer where editing was performed. Th is file was then transferred to an IBM 360/91 where management of the data base is currently maintained. The Statistical Analysis System (SAS) ${ }^{2}$ was the main computer software used for producing the microfiche output included in the pocket insert.

\section{DATA BASE USE}

The data base was prepared to allow for manipulation and display of any of the variables. Some of the manipulations include sorting, e.g., sorting the data base by manganese concentration, subsetting, e.g., separating those observations where a specific water type and/or lifestage are desired, and merging, e.g., adding new observations to the file.

A specific example manipulation of the data base is shown in Table 8. Here we show an entire program listing, less computer-installationspecific job control statements, of statments written in the SAS language, and a portion of the corresponding output, used to subset the data base for a group of organisms (crustaceans). The syntax of this language uses semicolons to end statements. Thus the code which precedes each semicolon constitutes a single instruction. This particular example creates a data set called EFFECTS. The variables are then defined, using mnemonic names, as they occur in columns of the

2A. J. Barr, J. H. Goodnight, J. P. Sall, and J. T. Helwig. 1976. A User's Guide to SAS 76. SAS Institute, Inc., P. 0. Box 10522, Raleigh, NC. 
input cards. Variables with character, rather than numeric, values are defined by a dollar sign on the input definition statement. The set of "IF" statements is used to include the observations with the desired organisms. A title card is used to label the printed output. This example illustrates how the data base can be subset for a particular organism to determine the effects, compounds, and test conditions which have been observed and included in the data base.

The example presented in Table 8 requires a set of data in the form described by the input statements. If the format changes for additional data, programs will have to be devised to manipulate the new format and merge the old and new data. The data base presented in the pocket insert has a format as described in the SAS input statements. A user who might be interested in manipulating the data base or implementing the data base on his computer should initially contact the third author for the availability of the data base and its available form.

In summary, our data base is an initial step towards expediting the tedious job of extracting, synthesizing, and tabulating, in numeric form, the current data available on toxic effects to aquatic organisms from trace elements in coal conversion process effluents. We feel this kind of effort is important to understanding the state-of-the-art with respect to inorganic toxic substances in aquatic systems. We emphasize the flexibility and ease of use of the computer methodology which we have developed for manipulating and displaying the data base. Researchers will find that the data base will ease the burden of literature search and evaluation. It will provide a starting point for directing researchers to current publications in toxic effects work and also provide a baseline for future research efforts. 
-Table 1. Elements included in the toxicity data base

\begin{tabular}{|c|c|c|}
\hline $\mathrm{Ag}$ (silver) & & In (manganese) \\
\hline AT (aruminum) & & 10 (mo lybdenum) \\
\hline As (arsenic) & & i (nickel) \\
\hline $\mathrm{Au}$ (gold) & & $b$ (lead) \\
\hline B (boron) & $\mathrm{Rb}$ & b (rubidium) \\
\hline Ba (barium) & Sb & b (antimony) \\
\hline Be (beryllium) & $\mathrm{Se}$ & e (selenium) \\
\hline $\mathrm{Br}$ (bromine) & Sn & $n(\operatorname{tin})$ \\
\hline Cd (cadmium) & Sr & $r$ (strontium) \\
\hline Ce (cerium) & & $h$ (thorium) \\
\hline Lo (cobalt) & $T 1$ & 1 (t1tantuili) \\
\hline $\mathrm{Cr}$ (chromium) & $\mathrm{TI}$ & 1 (thal $1 \mathrm{i} u m)$ \\
\hline Cu (copper) & $U$ & (uranium) \\
\hline Fe (1ron) & v & (vanadiunII) \\
\hline $\mathrm{Hg}$ (mercury) & & (tungsten) \\
\hline La (1 anthanum) & $\mathrm{Zn}$ & $n(z i n c)$ \\
\hline Li (lithium) & & $r$ (zirconium) \\
\hline $\mathrm{Mg}$ (magnesium). & & \\
\hline
\end{tabular}


Table 2. Variables and variable definitions used in the data base.

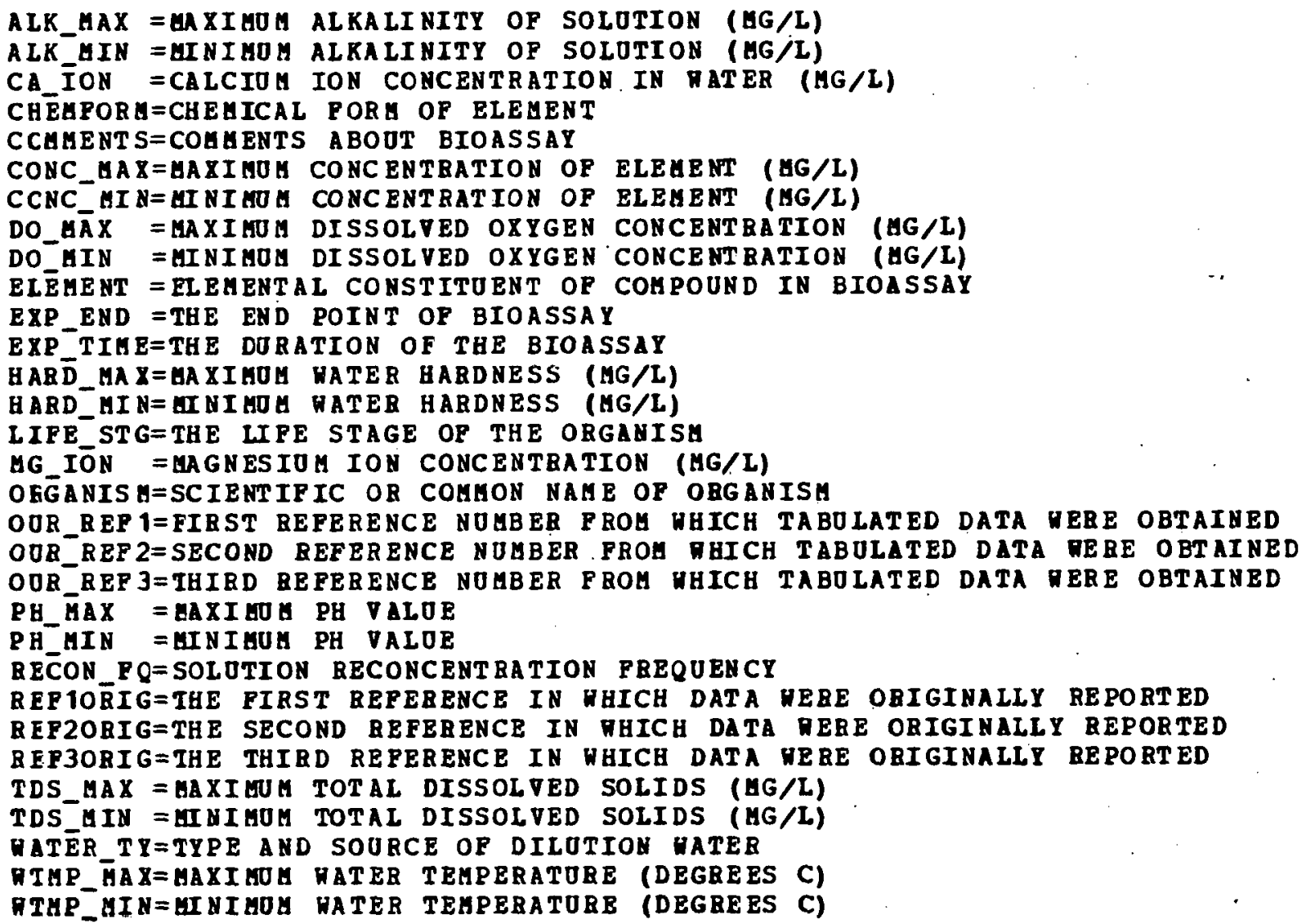


Table 3. Frequency cf occurrence of water chemistry and test conditions variables for each element. Each value represents the percent of the $N$ observations (listed under column heading $N$ ) for which data were reported for each of the elements and listed varlables.

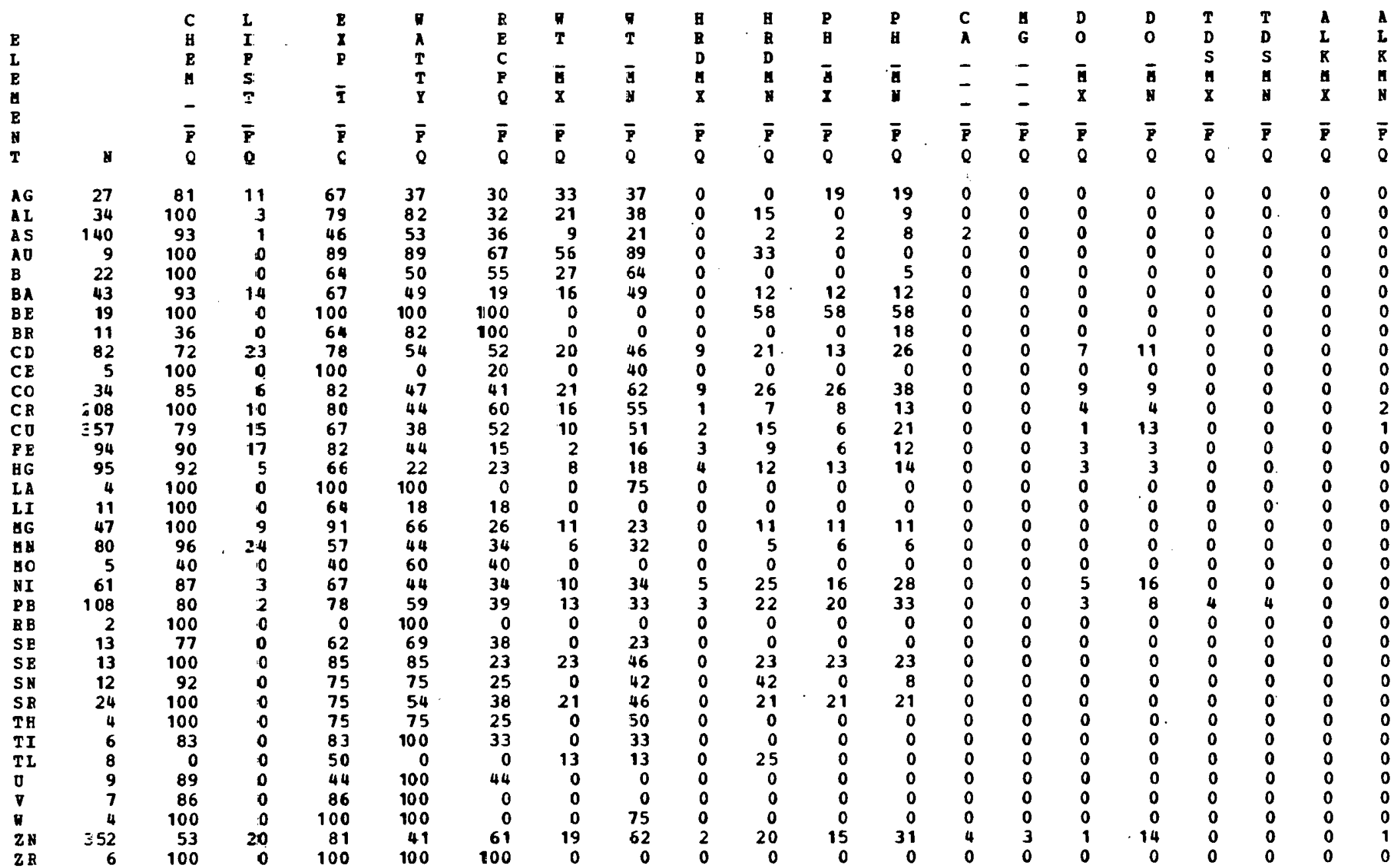


Table 3 (continued). Definitions of variables. used in the frequency-of-occurrence table, representing percent frequency of non-blank data.

Column Heading

Variable (see Table 2)

ALKMN_FQ

ALK MIN

ALKMX_FQ

- ALK MAX

$C A \quad F Q$

CA ION

$\mathrm{CHFM} \quad \mathrm{FQ}$

CHEMFORM

DO MN_FQ

DO_MIN

- DO_MX_FQ

DO_MAX

EXP_T_FQ

EXP_TIME

HRDMN FQ

HARD MIN

HRDMX_FQ

HARD MAX

LIFST_FQ

LIFE_STG .

MG

FQ

MG ION

$\mathrm{PH}$ MN FQ

PH_MIN

$\mathrm{PH} M \mathrm{MX} F \mathrm{FQ}$

PH MAX

RECFQ_FQ

RECON_FQ

TDSMN FQ

TDS MIN

TDSMX FQ

TDS MAX

WATTY_FQ

WATER TY

WT MN_FQ

WTMP MIN

WT MX_FQ

WTMP MAX 
Table 4. Sample page from data base.

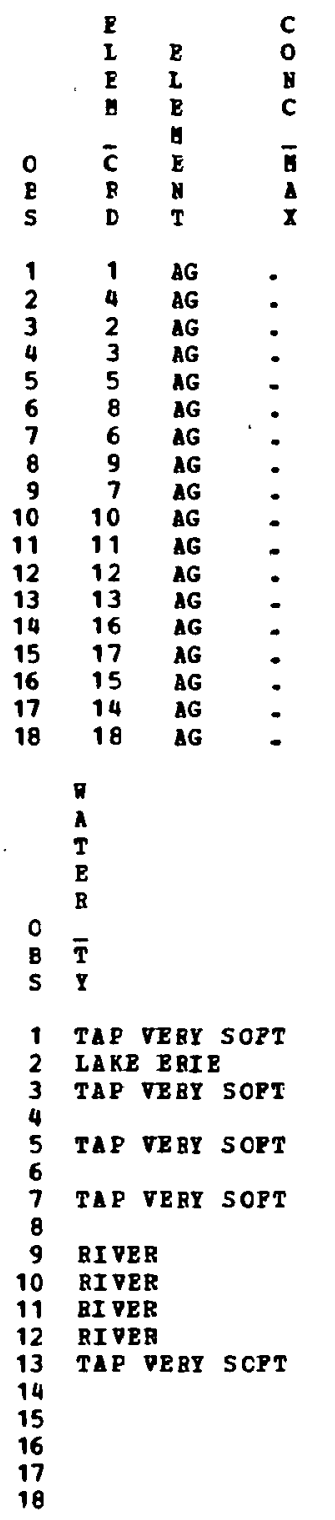

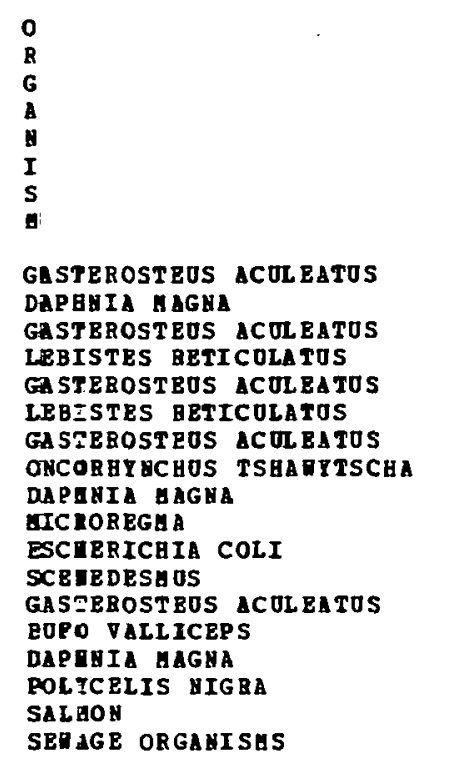

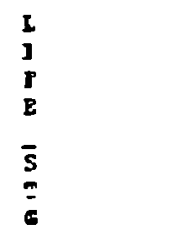

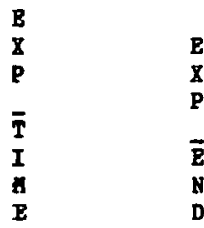

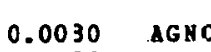

AGNO3

C.0070 AGNO3

0.0043 AGNO3

0.0130 AGNO3

C. 0120

AGHO3

$\begin{array}{ll}0.0250 & \\ 0.0250 & \text { AGHOS }\end{array}$

AGH.J3
AGHDJ 3

$\begin{array}{ll}\text { C. } 0330 & \text { AGHD3 } \\ \text { C.0330 } & \text { AGHO3 }\end{array}$

C.04DO AGEDS

C.05J0 AGNDJ
0.1030
C.1000

0.1000

c. 1050

AGHO3

C. 2800

AGHO3

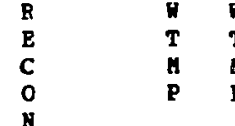

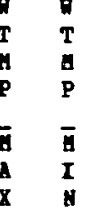

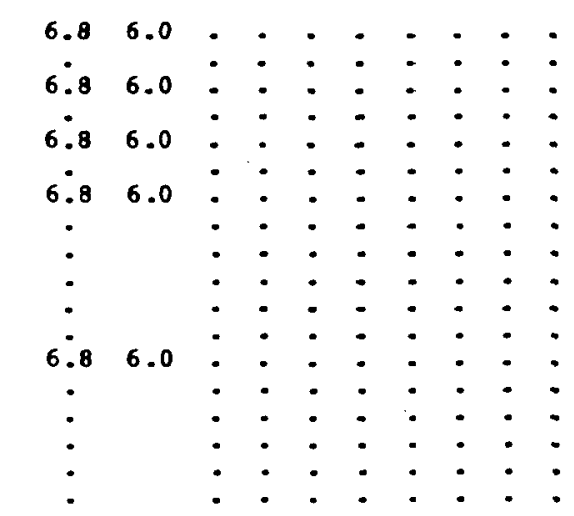

$\mathrm{Z}$
$\mathrm{X}$
$\mathrm{P}$
$\overline{\mathrm{E}}$
$\mathrm{N}$
$\mathrm{D}$ \begin{tabular}{c}
$\overline{8}$ \\
D \\
D \\
\hline
\end{tabular} ILI LETHAL

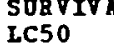
SORTIVAL ETHAL

SURVIVAL

PARTLi leteal

ILL

$48 \mathrm{~dB}$

96 HR

96 \&

96 HR

TADPOL ES

ILL

ILL ILI

SORVIVA
LETHAL

LETHAL

ILL

LET BAL

?RY $\quad 48$ HR

50X B. O.C. BEDUCTION

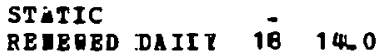

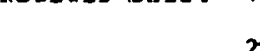

$$
\begin{array}{ll}
25 & 230 \\
2.7 & 23.0
\end{array}
$$

$27 \quad 230$

\begin{tabular}{|c|c|c|c|}
\hline $\begin{array}{l}\mathrm{R} \\
\mathrm{R} \\
\mathrm{P}\end{array}$ & $\begin{array}{l}\mathbf{R} \\
\mathbf{R} \\
\mathbf{P}\end{array}$ & & $\begin{array}{l}0 \\
0 \\
B\end{array}$ \\
\hline $\begin{array}{l}\mathbf{O} \\
\mathbf{R} \\
\mathbf{I} \\
\mathbf{G}\end{array}$ & $\begin{array}{l}2 \\
C \\
\text { R } \\
\text { I } \\
\text { G }\end{array}$ & 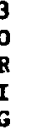 & \\
\hline 117 & 118 & & \\
\hline & 11 & & 159 \\
\hline 218 & & & $\begin{array}{l}75 \\
159\end{array}$ \\
\hline $\begin{array}{l}117 \\
217\end{array}$ & 118 & & 75 \\
\hline $\begin{array}{l}217 \\
117\end{array}$ & 119 & & 126 \\
\hline 153 & & & $\begin{array}{l}75 \\
75\end{array}$ \\
\hline 3 & & & 159 \\
\hline $\begin{array}{l}36 \\
35\end{array}$ & & & 159 \\
\hline $\begin{array}{l}35 \\
35\end{array}$ & & & 159 \\
\hline 117 & 118 & & 75 \\
\hline & & & 126 \\
\hline $\begin{array}{l}217 \\
117\end{array}$ & & & $\begin{array}{l}126 \\
159\end{array}$ \\
\hline 5. & & & $\begin{array}{l}159 \\
159\end{array}$ \\
\hline 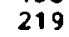 & & & 126 \\
\hline
\end{tabular}

QED DAIET 18 14.0 STATIC
ST ITIC 
Table 5. Scientific and common names of organisms

in toxicity table.

Fish

Abramis brama (bream)

Anguilla japonica (Japanese eel)

Anguilla rostrata (American eel)

Brachydanio rerio (zebra fish)

Carassius auratus (goldfish)

Carassius carassius (European goldfish, European carp)

Cyprinus carpio (carp)

Fundulus diaphanus (banded killifish)

Fundulus heteroclitus (mumichog)

Gambusia affinis (mosquitofish)

Gasterosteus aculeatus (threespine stickleback)

Gobio gobio (gudgeon, gobie)

Hesperoleucus (roach)

Ictalurus nebulosus (brown bullhead)

Ictalurus punctatus (channel catfish)

Lebistes reticulatus (guppy)

Lepomis cyanellus (green sunfish)

Lepomis gibbosus (pumpkinseed)

Lepomis macrochirus (bluegill)

Micropterus dolomieui (smallmouth bass)

Micropterus salmoides (1argemouth bass)

Morone americanus (white perch)

Morone saxatilis (striped bass, rockfish)

Nemachilus barbatulus (European stoneloach)

Notemigonus crysoleucas (golden shiner)

Notropis cornutus (common shiner)

Notropis hudsonius (spottail shiner)

Oncorhynchus gorbusca (pink salmon)

Oncorhynchus keta (chum salmon)

Oncorhynchus kisutch (coho salmon)

Oncorhynchus nerka (sockeye.sa 1mon)

Oncorhynchus tshawytscha (chinook salmon)

Orizias latipes (Japanese medaka)

Perca flavescens (yellow perch)

Perca fluviatilis (Eurasian perch)

Petromyzon martnus (sea lamprey)

Phoxinus phoxinus (minnow, red-sided shiner)

Pimephales promelas (fathead minnow)

Pomoxis (crappie)

Pungitius pungitius (ninespine stickleback)

Rutilus rutilus (roach)

Salmo clarki (cutthroat trout)

Salmo gairdnerii (rainbow or steelhead trout)

Salmo salar (AtTantic salmon)

Salmo trutta (brown trout)

Salvelinus fontinalis (brook trout)

Salvelinus namaycush (lake trout)

Scardinius erythrophthalmus (rudd)

Semotilus atromaculatus (creek chub)

Tilapia mossambica (mozambique mouthbrooder).

Tinca tinca (tench)

Xiphophorus maculatus (southern platyfish) 
Table 5. (Continued)

Protozoa

Euglena gracilis

Lepocinclis steinij

Microregma heterostoma

Paramecium

Uroglena

\section{Algae}

Anabacna circinal is

Anabaena flos-aqua

Anabaena variabilis

Anarystís

Ankistrodesmus

Asterionella

Calothrix

Cladophora glomerata

Chlamydomonas

Chlorella pyrenoidosa

chlorella sorokiniana

Chlorella variegata

Chlorella vulgaris

Chlorococcum humicola

Clathrocystes

Closterium

Cylindrospermum licheniforme

Gloeotrichia echinulata

Gomphonema parvulum

Hydrodictyon

Microcystis aeruginosa

Mirrospnra

Mougeotia

Navicula seminulum

Nitzschia linearis

$\checkmark$

Nitzschla ualed

Nostoc

Ocdogonium

oocystis

Oscillatoria

Pandorina

Phormidiuml ambiguum

Phormidium inundatum

Pithophora

Plectonema

scenedesmus obliquus

Scenedesmus quadricauda

Selanestrum capricornutum

Spirogyra

Stigeocionium tenue

Synedra

Synura

Tribonema

Ulothrix

Vaucheria

Zygnema 


\section{Table 5. (Continued)}

Other Plants

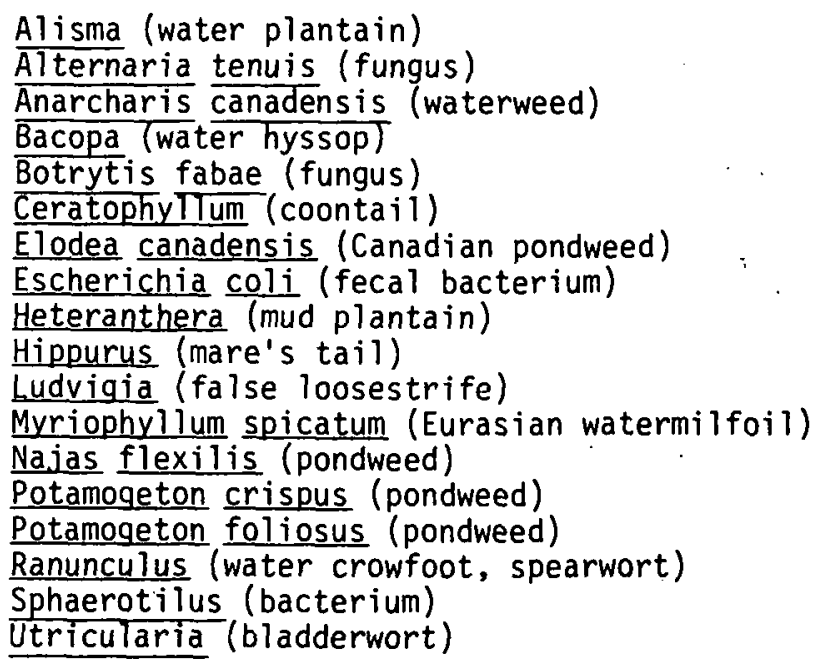

Alisma (water plantain)

Alternaria tenuis (fungus)

Anarcharis canadens is (waterweed)

Bacopa (water hyssop)

Botrytis fabae (fungus)

Ceratophyllum (coontail)

Elodea canadensis (Canadian pondweed)

Escherichia coli (fecal bacterium)

Heteranthera (mud plantain)

Hippurus (mare's tail)

Ludvigia (false loosestrife)

Myriophyllum spicatum (Eurasian watermilfoil)

Najas flexilis (pondweed)

Potamogeton crispus (pondweed)

Potamogeton foliosus (pondweed)

Ranunculus (water crowfoot, spearwort)

Sphaerotilus (bacterium)

Utricularia (bladderwort)

\section{Crustacea}

Asellus aquaticus (isopod)

Asellus communis (isopod)

Cyclops vernalis (copepod)

Daphnia Tongispina (cladoceran)

Daphnia magna (cladoceran)

Daphnia pulex (cladoceran)

Diaptomus oregonensis (copepod)

Gammarus lacustris (amphipod)

Gammarus pseudolimnaeus (amphipod)

Gammarus pulex (amphipod)

Hyalella knickerbockeri (amphipod)

Leptodora kindtii (cladoceran)

Mesocyclops leuckarti (copepod)

orconectes rusticus (decapod)

Simncenhalus serrulatus (c ladoceran)

\section{Insecta}

Acroneuria 1ycorias (stonefiy)

Argia (damselfly)

Caenis diminuta (mayfly)

Callibaetis (mayfly)

Chloeon simile (mayfly)

Ephemerella subvaria (mayfly)

Hydropsyche betteni (caddisfly)

Ischnura verticalis (damselfly)

Laccophilus maculosus (predaceous diving beetle)

Libellula (dragonfly)

Pteronarcys californica (stonefly)

Stenonema rubrum (mayfly)

Tendipes decorus (midge) 
Table 5. (Continued)

Mollusca

Ancylastrum fluviatile

Australorbis glabratus

Biomphalaria alexandrina

Biomphalaris biossyi

Bulinus contortus

Bulinus truncatus

Campe Toma decisum

Gyraulus circumstriatus

Helisoma companulata

Lymnaea palustris

Planorbis glabratus

Physa heterostropha

Physa integra

\section{Other Animals}

Bufo valliceps (frog)

l.imnodrilus hoffmeisteri (oligochaete)

Nais (oligochaete)

Polycelia nigra (planarian)

Rana pipiens (frog)

Xenopus laevis (toad) 
Táble 6. Cherrical form symbols as they appear on the microfiche and the corresponding chemical formulas.

\begin{tabular}{|c|c|c|c|}
\hline $\begin{array}{l}\text { PRINTOUT } \\
\text { SYMBOL }\end{array}$ & $\begin{array}{l}\text { CHEMICAL } \\
\text { FORMULA }\end{array}$ & $\begin{array}{l}\text { PRINTOUT } \\
\text { SYMBOL }\end{array}$ & $\begin{array}{l}\text { CHEMICAL } \\
\text { FORMULA }\end{array}$ \\
\hline & & . & \\
\hline AGNO3 & $\mathrm{AgNO}_{3}$ & LAAC3 & $\mathrm{LaAc}_{3}$ or $\mathrm{La}\left(\mathrm{C}_{2} \mathrm{H}_{3} \mathrm{O}_{2}\right)_{2}$ \\
\hline $\mathrm{AL}(\mathrm{NO} 03) 3$ & $\mathrm{~A} 1\left(\mathrm{NO}_{3}\right)_{3}$ & LICL & $\mathrm{LiCl}$ \\
\hline ALCL3 & $\mathrm{AlCl}_{3}$ & LIF & LiF \\
\hline $\mathrm{ALK}(\mathrm{SO} 4) 2$ & $\mathrm{AlK}\left(\mathrm{S}_{4}\right)_{2}$ & MEHG & $\mathrm{MeHq}$ or $\mathrm{CH}_{3} \mathrm{Hg}$ \\
\hline ALNH4 (SO4) 2 & $\mathrm{~A}_{1} \mathrm{NH}_{4}\left(\mathrm{SO}_{4}\right)_{2}$ & MEHGCL & $\mathrm{MeHgCl}$ or $\mathrm{CH}_{3} \mathrm{HgCl}$ \\
\hline $\mathrm{AL} 2(\mathrm{SO} 4) 3$ & $\mathrm{Al}_{2}\left(\mathrm{SO}_{4}\right)_{3}$ & $M G(N 03) 2$ & $\mathrm{Mg}\left(\mathrm{NO}_{3}\right)_{2}$ \\
\hline $\mathrm{ASO}_{-}^{-}$ & $\mathrm{AsO}_{2}^{-}$ & MGCL 2 & $\mathrm{MgCl}_{2}$ \\
\hline ASO4-3 & $\mathrm{AsO}_{4}^{-3}$ & MGNO3 & $\mathrm{MgNO}_{3}$ \\
\hline AS203 & $\mathrm{As}_{2} \mathrm{O}_{3}$ & MGSIF6 & $\mathrm{MgSiF}_{6}$ \\
\hline $\mathrm{BA}(\mathrm{N} 03) 2$ & $\mathrm{Ba}\left(\mathrm{NO}_{3}\right)_{2}$ & MGSO4 & $\mathrm{MgSO}_{4}$ \\
\hline BACL2 & $\mathrm{BaCl}_{2}$ & $\mathrm{MN}(\mathrm{NO} 3) 2$ & $\mathrm{Mn}\left(\mathrm{NO}_{3}\right)_{2}$ \\
\hline BAF2 & $\mathrm{BaF}_{2}{ }^{2}$ & MNCL2 & $\mathrm{MnCl}_{2}$ \\
\hline $\mathrm{BE}(\mathrm{N} 03) 2$ & $\mathrm{Be}\left(\mathrm{NO}_{3}\right)_{2}$ & MNCL3 & $\mathrm{MnCl}_{3}$ \\
\hline BECL2 & $\mathrm{BeCl}_{2}$ & MNF2 & $\mathrm{MnF}_{2}$ \\
\hline BESO4 & $\mathrm{BeSO}_{4}$ & MNSO4 & $\mathrm{MnSO}_{4}$ \\
\hline BF3 & $\mathrm{BF}_{3}$ & M003 & $\mathrm{MoO}_{3}$ \\
\hline $8407=$ & $\mathrm{B}_{4} \mathrm{O}_{7}^{-2}$ & NAASO2 & $\mathrm{NaAsO}_{2}$ \\
\hline $\mathrm{CD}(\mathrm{N} 03) 2$ & $\mathrm{Cd}\left(\mathrm{NO}_{3}\right)_{2}$ & NABO3 & $\mathrm{NaBO}_{3}$ \\
\hline COCL2 & $\mathrm{CdCl}_{2}$ & NABR & $\mathrm{NaBr}$ \\
\hline CDSO4 & $\mathrm{CdSO}_{4}^{\circ}$ & NABR03 & $\mathrm{NaBrO}_{3}$ \\
\hline CECL3 & $\mathrm{CeCl}_{3}$ & NA2ASO4 & $\mathrm{Na}_{2} \mathrm{AsO}_{4}$ \\
\hline CE2 (SO4) 3 & $\mathrm{Ce} 2\left(\mathrm{SO}_{4}\right)_{3}$ & NA2B407 & $\mathrm{Na}_{2} \mathrm{~B}_{4} \mathrm{O}_{7}$ \\
\hline $\mathrm{CO}(\mathrm{NO} 3)_{2}$ & $\mathrm{Co}\left(\mathrm{NO}_{3}\right)_{2}$ & NA2CRO4 & $\mathrm{Na}_{2} \mathrm{CrO}_{4}$ \\
\hline COCL & $\mathrm{CoCl}$ & NA2CR207 & $\mathrm{Na}_{2} \mathrm{Cr}_{2} \mathrm{O} 7$ \\
\hline COCL2 & $\mathrm{CoC} 12$ & NA2HASO4 & $\mathrm{Na}_{2} \mathrm{HA}_{5} \mathrm{O}$ \\
\hline COSO4 & $\mathrm{CoSO}_{4}$ & NA2SE03 & $\mathrm{Na}_{2} \mathrm{SeO}_{3}$ \\
\hline CRCL3 & $\mathrm{CrCl}_{3}$ & NA2W04 & $\mathrm{Na}_{2}^{2} \mathrm{WO}_{4}$ \\
\hline CRO3 & $\mathrm{CrO}_{3}$ & NA3AS04 & $\mathrm{Na}_{3} \mathrm{~A}_{5} \mathrm{O}_{4}$ \\
\hline CR04 $=$ & $\mathrm{CrO}_{4}^{-2}$ & (NH4) 2CRO4 & $\left(\mathrm{NH}_{4}\right)_{2} \mathrm{CrO}_{4}$ \\
\hline $\mathrm{CR} 2\left(\mathrm{SO}_{4}\right) 3$ & $\mathrm{Cr}_{2}(\mathrm{SO} \eta)_{3}$ & $(\mathrm{NH} 4) 2 \mathrm{CR} 207$ & $\left(\mathrm{NH}_{4}\right)_{2} \mathrm{Cr}_{2} \mathrm{O}_{7}$ \\
\hline $\mathrm{CR} 207=$ & $\mathrm{Cr}_{2} \mathrm{O}_{7}-2$ & $\mathrm{NI}(\mathrm{NO3}) 2$ & $\mathrm{Ni}\left(\mathrm{NO}_{3}\right)_{2}$ \\
\hline $\mathrm{CU}(\mathrm{N} 03) 2$ & $\mathrm{Cu}\left(\mathrm{NO}_{3}\right)_{2}$ & NICL2 & $\mathrm{NiCl}_{2}$ \\
\hline CUCL2 & $\mathrm{CuCl}_{2}$ & NISO4 & $\mathrm{NiSO}_{4}$ \\
\hline CUSO4 & $\mathrm{CuSO}_{4}^{2}$ & $\mathrm{~PB}(\mathrm{NO} 3) 2$ & $\mathrm{~Pb}\left(\mathrm{NO}_{3}\right)_{2}$ \\
\hline FECL2 & $\mathrm{FeCl}_{2}$ & PBAC2 & $\mathrm{PbAC} 2$ or $\mathrm{Pb}\left(\mathrm{C}_{2} \mathrm{H}_{3} \mathrm{O}_{2}\right)_{2}$ \\
\hline FECL3 & $\mathrm{FeCl}_{3}$ & PBCL2 & $\mathrm{PbCl} 2$ \\
\hline FEO & $\mathrm{FeO}$ & PBSO4 & $\mathrm{PbSO}_{4}$ \\
\hline FES & FeS & PBO & $\mathrm{PbO}$ \\
\hline FESO3 & $\mathrm{FeSO}_{3}$ & PHENYL HLOHH & Phenyl $\mathrm{HgOH}$ or $\mathrm{C}_{6} \mathrm{H}_{6} \mathrm{UHg}$ \\
\hline FESO4 & $\mathrm{FeSO}_{4}$ & PHENYL HGAC & Phenyl $\mathrm{HgAc}$ or $\mathrm{C}_{8} \mathrm{H}_{8} \mathrm{O}_{2} \mathrm{Hg}$ \\
\hline $\mathrm{FE2}(\mathrm{SO4}) 3$ & $\mathrm{Fe}_{2}\left(\mathrm{SO}_{4}\right)_{3}$ & PHENYL HGN03 & Phenyl $\mathrm{HgNO}_{3}$ or $\mathrm{C}_{12} \mathrm{H}_{11} \mathrm{OHgNO}_{3}$ \\
\hline FE203 & $\mathrm{Fe}_{2} \mathrm{O}_{3}$ & $\mathrm{RBCL}$ & $\mathrm{RbCl}$ \\
\hline HAUCL 4 & $\mathrm{HAuCl}_{4}$ & SBCL3 & $\mathrm{SbCl}_{3}$ \\
\hline $\mathrm{HBO3}$ & $\mathrm{HBO}_{3}$ & SBF3 & $\mathrm{SbF}_{3}$ \\
\hline $\mathrm{H} 3 \mathrm{BO} 4$ & $\mathrm{H}_{3} \mathrm{BO}_{4}$ & SBKTARTRATE & SbKTartrate or $\mathrm{KSbOC}_{4} \mathrm{H}_{4} \mathrm{O}_{6}$ \\
\hline $\mathrm{HG}(\mathrm{NO3}) 2$ & $\mathrm{Hg}\left(\mathrm{NO}_{3}\right)_{2}$ & SE02 & $\mathrm{SeO}_{2}$ \\
\hline HGAC2 & $\mathrm{Hg} \mathrm{C}_{2}$ or $\mathrm{Hg}\left(\mathrm{C}_{2} \mathrm{H}_{3} \mathrm{O}_{2}\right)_{2}$ & SE03-2 & $\mathrm{SeO}_{3}^{-2}$ \\
\hline HGCL2 & $\mathrm{HgCl}_{2}$ & SNCL2 & $\mathrm{SnCl}_{2}$ \\
\hline HGNO2 & $\mathrm{HgNO}_{2}$ & SNCL4 & $\mathrm{SnCl}_{4}$ \\
\hline HGSO 4 & $\mathrm{HgSO}_{4}$ & $\mathrm{SR}(\mathrm{NO} 3) 2$ & $\mathrm{Sr}\left(\mathrm{NO}_{3}\right)_{2}$ \\
\hline $\mathrm{KCR}(\mathrm{SO} 4) 2$ & $\mathrm{KCr}\left(\mathrm{SO}_{4}\right)_{2}$ & SRCL2 & $\mathrm{SrCl}_{2}$ \\
\hline KCR207 & $\mathrm{KCr}_{2} \mathrm{O}_{7}$ & SRF2 & $\mathrm{SrF}_{2}$ \\
\hline KMNO4 & $\mathrm{KMnO}_{4}$ & $\mathrm{TH}(\mathrm{NO} 3) 4$ & $\mathrm{Th}\left(\mathrm{NO}_{3}\right)_{4}$ \\
\hline K2CRO4 & $\mathrm{K}_{2} \mathrm{CrO}_{4}$ & THCI 4 & ThC. 14 \\
\hline $\mathrm{K} 2 \mathrm{Cr} 207$ & $\mathrm{~K}_{2} \mathrm{Cr}_{2} \mathrm{O}_{7}$ & TICL2 & $\mathrm{TiCl}_{2}$ \\
\hline
\end{tabular}


Table 6. (Continued)

\begin{tabular}{|c|c|}
\hline $\begin{array}{l}\text { PRINTOUT } \\
\text { SYMBOL } \\
\end{array}$ & $\begin{array}{l}\text { CHEMICAL } \\
\text { FORMULA }\end{array}$ \\
\hline $\begin{array}{l}\text { TI2(SO4)3 } \\
\text { UO2(NO3)2 } \\
\text { UO2AC2 } \\
\text { UO2SO4 } \\
\text { VOSO4 } \\
\text { V2O5 } \\
\text { ZN(NO3)2 } \\
\text { ZNCL2 } \\
\text { ZNSO4 } \\
\text { TR (SO4)2 } \\
\text { ZROCL2 }\end{array}$ & $\begin{array}{l}\mathrm{Ti}_{2}\left(\mathrm{SO}_{4}\right)_{3} \\
\mathrm{VO}_{2}\left(\mathrm{NO}_{3}\right)_{2} \\
\mathrm{UO}_{2} \mathrm{Ac}_{2} \text { or } \mathrm{UO}_{2}\left(\mathrm{C}_{2} \mathrm{H}_{3} \mathrm{O}_{2}\right)_{2} \\
\mathrm{UO}_{2} \mathrm{SO}_{4} \\
\mathrm{VOSO}_{4} \\
\mathrm{~V}_{2} \mathrm{O}_{5} \\
\mathrm{Zn}\left(\mathrm{NO}_{3}\right)_{2} \\
\mathrm{ZnCl}_{2} \\
\mathrm{ZnSO}_{4} \\
\mathrm{Zr}\left(\mathrm{SO}_{4}\right)_{2} \\
\mathrm{ZrOCl} l_{2}\end{array}$ \\
\hline
\end{tabular}


Table 7. List of references used to document the data presented in the toxicity data base. (Entire list of references also appears on microfiche in pocket insert on inside back cover.)

1. Abegg, R. 1950. Some effects of inorganic salts on the blood specific gravity and tissue fluids of the Bluegill, Lepomis . macrochirus Raf. Physiol. Zool. 23:124-134.

2. Affleck, A. J. 1952. Zinc poisoning in a trout hatchery. Austr. J. Mar. Freshwater Res. 3:142.

3. Alabaster, J. S. 1956. The toxicity of certain weed killers to trout. Proc. 3rd Brit. Weed Contr. Conf. 2:807-808.

4. Amend, D. F., W. T. Yasutake, and R. Morgan. 1969. Trans. Amer. Fish. Soc. 98:419.

5. Anderson, B. G. 1944. The toxicity thresholds of various substances found in industrial wastes as determined by the use of Daphnia magna. Sewage Works J. 16:1156.

6. Anderson, B. G. 1946. The toxicity thresholds of various sodium salts determined by the use of Daphnia magna. Sewage Works J. $18(1): 82-87$.

7. Anderson, B. G. 1948. The apparent thresholds of toxicity of Daphnia magna for chlorides of various metals when added to Lake Erie water. Trans. Amer. Fish. Soc. 78:96.

8. Anderson, B. G., D. C. Chandler, T. F. Andrews, and W. J. Jahoda. 1948. The evaluation of aquatic invertebrates as assay organisms for the determination of the toxicity of industrial wastes. Amer. Petrol. Inst. Project Final Rept. 51.

9. Anonymous. 1950. Ohio River Valley Water Sanitation Commission, Sub-committee on Toxicities, Metal Finishing Industries Action Committee. Report No. 3.

10. Anonymous. 1956. The relationship of body size of the bluegill sunfish to the acute toxicity of some common chemicals. Phila. Acad. Sci. Mimeo.

11. Anonymous. 1958. Water pollution research for 1957. Water Pollut. Res. Bd., Dept. of Sci. Ind. Res., H. M. Stationery Office, London.

12. Anonymous. 1958. Waste waters and fish. Dept. of Sci. Ind. Res. (Eng.) Notes on water pollution 2. 
13. Anonymous. 1960. Toxic effects of organic and inorganic pollutants on young salmon and trout. State of Washington, Dept. Fisheries Res. Bull. No. 5.

14. Anonymous. 1960: The Merck index of chemicals and drugs. 7th ed.

15. Anonymous. 1960. Report of the Water Pollution Research Board, with the report of the director of the Water Pollution Research Laboratory for the year 1959. Dept. of Sci. Ind. Res., H. M. Stationery Office, London.

16. Annonymous. 1960. The sersitivity of aquatic life to certain chemicals commonly found in industrial wastes. Acad. Nat. sci., thila.

17. Applegate, V. C., J. H. Howell, A. E. Hall, and M. A. Smith. Toxicity of 4,346 chemicals to larval lampreys and fishes. Fish and Wildlife Serv., Spec. Sci. Rept. Fish. $207: 157$.

18. Arthur, J. W. and E. N. Leonard. 1970. Effects of copper on Gammarus pseudol imnaeus, Physa integra, and Campeloma decisum in soft water. J. Fish. Res. Bd. Canada 27(7):1277-1283.

19. Bachmann, H., A. Birrer, and H. Weber. 1933. The poisonous effect of chemical substances on lower water organisms. Zeits. Hydrol. (Zurich) 6,63: Water Pollut. Abst. 9:1141 (1936).

20. Ball, I. R. 1967. The relative susceptibilities of some species of freshwater fish to poisons-II. Zinc. Water Res. $1(11 / 12): 777-783$.

21. Bandt, H. J. 1946. Intensified injurious effects on fish, especially the incrcaccd toxio effect produced by a rombination of sewage poisons, Beitr. Wass. Abwass., Fischereichem $1: 15$.

22. Bartlett, L., et al. 1974. Effects of copper, zinc, and cadmium on Selanestrum capricornutum, Water RPS. (G.R.) 8:179.

23. Batte, E. G., L. E. Swanson, and J. B. Murphy. 1951. New molluscicides for the control of freshwater snails. Amer. J. Vet. Res. 12(3):158-160.

24. Becker, C. D., and T. O. Thatcher. 1973. Toxicity of power plant chemicals to aquatic life. Battelle Pacific Northwest Laboratories, Richland, Washington. Wash-1249 UCll.

25. Belding, D. L. 1927. Toxicity experiments with fish in reference to trade waste pollution. Trans. Amer. Fish. Soc. $57: 100$.

26. Betzer, N. and Y. Kott. 1969. Effect of halogens on algae-II. Cladophora sp. Water Res. $3(4): 257-264$. 
27. Beyerly, G. B. and J. E. Williams. 1967. Attempted control of bluegill reproduction in lakes by the application of copper sulfate crystals to spawning nests. Prog. Fish cult. 29(3):150-155.

28. Biesinger, K. E. and G. M. Christensen. 1972. Effects of various metals on survival, growth, reproduction, and metabolism of Daphnia magna. J. Fish. Res. Bd. Canada $29(12): 1691-1700$.

29. Bijan, H. and R. Deschiens. 1956. Effect of barium salts on the mollusk vectors of schistosomiasis. Bull. Soc. Pat. Exot. 49:455.

30. Binet, $L$. and P. Nicolle. 1940. Influence of the salinity of the ambient medium on the toxicity of mercuric chloride for the stickleback. C. R. Soc. Biol. (Paris) 134:562.

31. Boetius, J. 1960-61. Lethal action of mercuric chloride and phenylmercuric acetate on fishes. Medd. Darm. Fiskeri-og Havunders, n.s., 3:93-115.

32. Bond, H. W. and M. O. Nolan. 1954. Results of laboratory screening tests of chemical compounds for molluscacidal activity. II. Compounds of mercury. Amer. J. Tropical Med. and Hyg. 3:187.

33. Boschetti, M. M. and T. F. McLoughlin. 1957. Toxicity of sodium arsenite to minnows. Sanitalk. 5(4):14-18.

34. Brice, S. 1973. The acute effects of methyl mercuric chloride on channel catfish (Ictalurus punctatus) fingerlings and their behavioral response to various chemícal stimuli. Thesis, Florida State Univ.

35. Bringmann, G. and R. Kuhn. 1959. The toxic effects of waste water on aquatic bacteria, algae, and small crustaceans. Gesundheits-Ing. 80:115-120.

36. Bringmann, G. and R. Kuhn. 1959. Water Toxicology studies with protozoans as test organisms. Gesundheits-Ing. 80:239-243.

37. Brown, F. M. 1949. Askern Lake, pp. 22-23. In: Report of the Naturalist's Union for 1948, Natural ist (London) No. 828.

38. Brown, V. B. 1968. The calculation of the acute toxicity of mixtures of poisons to rainbow trout. Water Res. $2(10): 723-733$.

39. Brown, V. M. and R. A. Dalton. 1970. The acute toxicity to rainbow trout of mixtures of copper, phenol, zinc and nickel. J. Fish. Biol. $2(2): 211-216$.

40. Brungs, W. A. 1969. Chronic toxicity of zinc to the fathead minnow, Pimephales promelas Rafinesque. Trans. Amer. Fish. Soc. $98(2): 272-279$. 
41. Brungs, W. A., E. N. Leonard, and J. M. McKim. 1973. Acute and long-term accumulation of copper by the brown bullhead, Ictalurus nebulosus. J. Fish. Res. Bd. Canada 30:583-586.

42. Buhler, D. R. 1973. Environmental contamination by toxic metals. In: Heavy Metals in the environmental. Seminar conducted by Water Resources Research Institute, Oregon State Univ.

43. Bumbu, Y. V. and A. S. Mokryak. 1973. Effect of some trace elements on the development of the algae Scenedesmus quadricauda. Izv. Akad. Nauk Mold. SSR, Ser. Biol. Khim. Nauk (USSR) 1.

44. Burgess, S, G. 1957. The analysis of trade-waste waters. In: The treatment of trade-waste waters and the prevention of river pollution. Kings College, Univ. of Durham, Newcastle-Upon-Tyne, England.

45. Burton, D. T., E. L. Morgan and J. Cairns, Jr. 1972. Mortality curves of bluegills (Lepomis macrochirus Rafinesque) simultaneously exposed to temperature and zinc stress. Trans. Amer. Fish. Soc. $101(3): 435-441$.

46. Cairns, J., Jr., T. K. Bahns, D. T. Burton, K. L. Dickson, R. E. Sparks, and W. T. Waller. 1972. The effects of $\mathrm{pH}$, solubility and temperature upon the acute toxicity of zinc to the bluegill suntish (Lepomis macrochirus Rafinesque). Trans. Kansas Acad. Sci. 74(1):81-92.

47. Cairns, J., Jr. and A. Schejer. 1958. The effects of temporature and hardness of water upon the toxicity of zins. to the pond snail, Physa heterostropha (Say). Notulae Natur., No. 308. 11 pp.

48. Calrns, J., Jr. and A. Scheier. 1958. The celationship of blucgill sunfish body size to tolerance for some common chemicals. Ind. Wastes $3: 5,126$.

49. Cairns, J., Jr and A. Scheier. 1959. The relationship of blueyill sunfish body size to tolerance for some common chemicals. Proc. 13th Ind. Waste Conf., Purdue Univ., Eng. Bull. . 43:3,243.

50. Cairns, J., Jr. and A. Scheier. 1959. The effects of temperature and hardness of water upon the toxicity of potassium dichromate to the common bluegill sunfish, pp. 86-97. In: Trans. N. E. Wildlife Conf., loth Ann. Meeting, Montreal, Quebec, Canada (Jan. 1958).

51. Cairns, J., Jr. and A. Scheier. 1968. A comparison of the toxicity of some common industrial waste components tested individually and combined. Prog. Fish-Cult. $30(1) 3-8$. 
52. Cairns, J., Jr., A. Scheier and J. J. Loos. 1965. A comparison of the sensitivity to certain chemicals of adult zebra danios Brachydanio rerio (Hamilton-Buchanan) and zebra eggs with that of adult bluegill sunfish Lepomis macrochirus Rafinesque. Notulae Natur., No. 381.9 pp.

53. Carpenter, K. E. 1925. On the biological factors involved in the destruction of river-fisheries by pollution due to lead-mining. Ann. Appl. Biol. 12:1-13.

54. Carpenter, K. E. 1927. The lethal action of soluble metallic salts on fishes. Brit. J. Exp. Biol. 4:378.

55. Carpenter, K. E. 1930. Further researches on the action of metallic salts on fishes. J. Exp. Zool. 56:407-422.

56. Chapman, G. 1973. Effects of heavy metals on fish. In: Heavy metals in the environment. Seminar conducted by Water Resources Research Institute, Oregon State Univ.

57. Chen, C. W. and R. E. Selleck. 1969. A kinetic model of fish toxicity threshold. J. Water Pollut. Control Fed. (Res. Suppl) $41(8):$ R294-R308.

58. Christensen, G. M., J. M. McKim, W. A. Brungs, and E. P. Hunt. 1972. Changes in the blood of the brown bullhead (Ictalurus nebulosus (Lesueur)) following short and long term exposure to copper (II). Toxicol. Appl. Pharm. $23: 417-427$.

59. Clemens, H. P. and K. E. Sneed. 1959. Lethal doses of several commercial chemicals for fingerling channel catfish. Special Scientific Rept. Fisheries No. 316, U.S. Dept. of Interior.

60. Cole, A. E. 194l. The effects of pollutional wastes on fish life. Symp. on Hydrobiol., Univ. of Wisconsin $: 241$.

61. Colmano, G 1973. Molybdenum toxicity: abnormal cell division of teratogenic appearance in Euglena gracilis. Bull. Envir. contam. Toxicol. 9:6.

62. Colorado Game, Fish and Parks Div. 1971. Water pollution studies: study of the effects of metallic ions on fish and aquatic organisms. Job Progr. Rept., Federal Aid Project F-33-R-6. 116 pp.

63. Cope, O. B. 1965. Sport fishery investigation. In: Effects of pesticides on fish and wildlife, 1964 findings of the Fish Wildlife Serv., U.S. Fish Wildlife Serv., Circ. 226:51-63.

64. Cope, O. B. 1966. Contamination of the fresh-water ecosystem by pesticides. J. Appl. Ecol. 3 (Suppl.):33-44.

65. Cowel1, B. C. 1965. The effects of sodium arsenite and silvex on the plankton populations in farm ponds. 'Trans. Amer. Fish. Soc. $94: 371-377$. 
66. Crance, J. H. 1963. The effects of copper sulfate on Microcystis and zooplankton in ponds. Prog. Fish-Cult. 25(4):198-202.

67. Crandall, C. A. and C. J. Goodnight. 1962. Effects of sublethal concentrations of several toxicants on the common guppy, Lebistes reticulatus. Limnol. Oceanogr. 7:233-239.

68. Crandall, C. A. and C. J. Goodnight. 1963. The effects of sublethal concentrations of several toxicants to the common guppy, Lebistes reticulatus. Trans. Amer. Microsc. Soc. $82: 59-7 \overline{3}$.

69. Crosby, D. G. and R. K. Tucker. 1966. Toxicity of aquatic herbicides to Daphnia maqna. Science 154:289-291.

70. Dawson, A. B. 1935. The hemopoietic response in the catfish, Ameiurus nebulosus, to chronic lead poisoning. Biol. Bull. 68:335-346.

71. Deschiens, R., V. Molinari, and D. Bertrand. 1957. The action of "zinc water" as a toxic agent on molluscs. Bull. Soc. Path. Exot. 50(1):59-61 (In French); Biol. Abst. $32,5811(1958)$.

72. Dilling, W. J. and C. W. Healey. 1926. Influence of lead and the metallic ions of copper, zinc, thorium, beryllium and thallium on. the germination of frogs' spawn and on the growth of tadpoles. Ann. Appl. Biol. 13(2):177-188.

73. Dilling, w. S., $\quad$ c. $w$. Healey, and $w$. C. Smith. 1926. Experiments on the effects of lead on the growth of plaice (Pleuronects platessa). Ann. Appl. Biol. 13:168-176.

74. Douoroff, P. 1952. Some recent developments in the study of toxic industrial wastes, pp. 21-25. In: Proc. 4th Ann. Pacific N.W. Ind. Waste Conf., Washington State Univ., Pullman.

75. Douoroff, P. and M. Katz. 1953. Critical review of literature

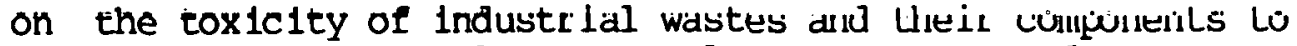
fish: II. The metals, ag anlta. Sewngr Ind. Wastes $25(7)$ : 802-839.

76. Dowden, B. and H. J. Bennett. 1965. Toxicity of selected chemicals to certain animals. J. Water Pollut. Control Fed. $37(9): 1308-1316$.

77. Dyk, V. 1942. Die widerstandsfahigkeit einiger Fische bei Wasserverunreinigung mit Ferrosulfat. Sbornik Ceskoslov. Akad. Zemedelske 17:450-454.

78. Eaton, J. G. 1973. Chronic toxicity of a copper, cadmium and zinc mixture to the fathead minnow (Pimephales promelas Rafinesque). Water Research 7:1723-1736. 
79. Ebeling, G. 1928. Uber die Giftigkeit einiger Schwermetallsalze an Hand eines Falles aus der Praxis. Zeits. Fischerei $26: 49-61$.

80. Effects of pollution on fish life, heavy metals. A literature review. 1970. J. Water pollut. Contr. Fed. $42(6)$ : $987-1002$.

81. Eipper, A. W. 1959. Effects of five herbicides on farm pond plants and fish. N.Y. Fish Game J. 6(1):45-56.

82. Ellis, M. M. 1937. Detection and measurement of stream pollution (related principally to fish life). U.S. Dept. of Commerce, Bur. of Fisheries Bull. 22.

83. Fairchild, E. J. 1955. Low dissolved oxygen. Effect upon the toxicity of certain inorganic salts to the aquatic invertebrate Daphnia magna. Louisiana State Univ. Eng. Expt. Sta. Bull. No. 51:95.

84. Fingal, W. and H. M. Kaplan. 1963. Susceptibility of Xenopus laevis to copper sulfate. Copeia 1963(1):155-156.

85. Fisher, A. 1956. The effect of copper sulfate on some microorganisms in fish ponds. Badidgeh, Bull. Fish Culture in Israel $8(2): 21-27$.

86. Fitzgerald, G. P. 1964. An evaluation of potassium permanganate as an algicide for water cooling towers. Ind. Eng. Chem. $56(2)$ : 79 . (Abstract).

87. Fitzgerald, G. P. and S. L. Faust. 1963. Factors affecting the algicidal and algistatic properties of copper. Appl. Microbiol. $11(4): 345-351$.

88. Fowler, J. R. 1931. The relations of numbers of animals to survival in toxic concentrations of electrolytes. Physiol. Zool. 4:214-245.

89. Freeman, L. and I. Fowler. 1953. Toxicity of combinations of certain inorganic compounds to Daphnia magna Straus. Sewage Ind. Wastes $25(10): 1191-1195$.

90. Fromm, P. O. and R. H. Schiffman. 1958. Toxic action of hexavalent chromium on largemouth bass. J. Wildlife Mgt. $22(1)$ : 40-44.

91. Fromm, P. O. and R. M. Stokes. 1962. Assimilation and elimination of chromium by trout. J. Water Pollut. Control Fed. $34(11): 1151-1155$.

92. Fujiya, M. 1961. Use of electrophoretic serum separation in fish studies. J. Water Pollut. Contr. Fed. 33:250.

93. Ghosh, M. M. and P. D. Zugger. 1973. Toxic effects of mercury on the activated sludge process. J. Water Pollut. Contr. Fed. 45:424. 
94. Gilderhus, P. A. 1966. Some effects of sublethal concentrations of sodium arsenite on bluegills and the aquatic environment. Trans. Amer. Fish Soc. $95(3): 289-296$.

95. Gill, J. M., J. H. Huguet, and E. A. Pearson. 1960. Submarine dispersal system for treated chenical wastes. J. Water Pollut. Contr. Fed. 32:858.

96. Gohar, H. A. F. and H. El-Gindy. 1961. Tolerance of vector snails of bilharziasis and fasciolasis to some chemicals. Proc. Egypt. Acad. Sci. 16:37-48.

97. Gooding, D. 1954. Pollution research, toxicity studies, p. 44. In: 64th Annual Report, Wash. State Dept. F'isheries, Olympia.

98. Goodman, J. R. 195i. Toxicity of zinc for rainbow trout (Salmo gairdnerii). Calif. Fish and Game 37:191-194.

99. Grande, M. 1967. Effect of copper and zinc on salmonid fishes, pp. 97-111. In: Advances in Water Pollution Research, Proc. 3rd Intl. Conf., Vol. I., Water Pollut. Control Fed., Washington, D.C.

100. Gregory, L. 1974. The effect of effluent components from chlor-alkali plants on aquatic organisms. A literature review. Fish. Res. Bd. Canada Tech. Rept. No. 228. 94 pp.

101. Grindley, J. 1946. Toxicity to rainbow trout and minnows of some substances known to be present in waste waters discharged to rivers. Ann. Appl. Biol. 33:103.

102. Grushko, Y. M. 1949. Toxic substances and the method of establishing hygienic standards for their permissible concentration in water reservoirs. Gigiena 7:11.

103. Haga, Y., H. Haga, T. Hagino, and T. Kariya. 1970. Studies on the postmortem identification of the pollutant in fish killed by water pollution-XII. Acute poisoning with mereury (1). Bull. Jap. Soc. Sci. Fish. 36:225-231.

104. Hale, F. E. 1937. The use of copper sulphate in control of microscopic organisms. Nichols Copper Co., New York. 35 pp.

105. Harry, H. W. and D. V. Aldrich. 1958. The ecology of Australorbis glabratus in Puerto Rico. Bull. of the World Health Organi zation $18(5-6): 819$.

106. Harry, H. W., B. G. Cumbie, and J. M. deJesus. 1957. Study on the quality of fresh waters of Puerto Rico relative to the occurrence of Australorbis glabratus. Amer. J. Trop. Med. Hygiene $6(2): 313-322$.

107. Hassall, K. A. 1962. A specific effect of copper on the respiration of Chlorella vulgaris. Nature 193:90. 


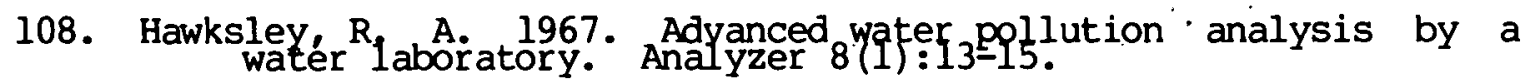

109. Hazel, C. R. and S. J. Meith. 1970. Bioassay of king salmon eggs and sac fry in copper solutions. Calif. Fish Game $56(2): 121-124$.

110. Hughes, J. S. 1973. Acute toxicity of thirty chemicals to striped bass (Morone saxatilis): Presented at the Western Asso. State Game Fish Commrs., Salt Lake City, Utah. 15 pp.

111. Hughes, J. S. and J. T. Davis. 1967. Effects of selected herbicides on bluegill sunfish, pp. 480-482. In: Proc. 18th Ann. Conf. Southeastern Assoc. Game Fish Commrs.

112. Hutchinson, G. E. 1933. Experimental studies in ecology. I. The magnesium tolerance of Daphniidae and its ecological significance. Internat. Rev. Hydrobiol. 28:90-108.

113. Ingols, R. S. 1955. Evaluation of toxicity. Sewage Ind. Wastes $27(1): 26-33$.

114. Ingols, R. S. 1956. Toxicity of copper and zinc ions in the dilution B.O.D. tests. Sewage Ind. Wastes $28(9): 1168-1169$.

115. Ingols, R. S. and R. H. Fetner. 1961. Toxicity of chromium compounds under aerobic conditions. J. Water Pollut. Contr. Fed. $33(4): 366-370$.

116. Iwao, T. 1936. Comparative investigation of the toxicity of various metlas. J. Exp. Pharmacol. (Jap.) 10:357-380.

117. Jones, J. R. E. 1938. The relative toxicity of salts of lead, zinc, and copper to the stickleback (Gasterosteus aculeatus L.) and the effect of calcium on the toxicity of lead and zinc salts. J. Exp. Biol. 15:394-407.

118. Jones, J. R. E. 1939. The relation between the electrolytic solution pressures of metals and their toxicity to the stickleback (Gasterosteus aculeatus L.). J. Exp. Biol. $16: 425-437$.

119. Jones, J. R. E. 1940. A further study of the relation between toxicity and solution pressure, with Polycel is nigra as test animal. J. Exp. Biol. 17:408.

120. Jones, J. R. E. 1941. A study of the relative toxicity of anions, with Polycelis nigra as test animal. J.' Exptl. Biol. 18:170-181.

121. Jones, J. R. E. 1957. Fish and river pollution. In: Aspects of river pollution, L. Klein, ed., Butterworths Sci. Publ., London.

122. Jones, J. R. E. 1964. Fish and river pollution. Washington: Butterworths. 203 pp. 


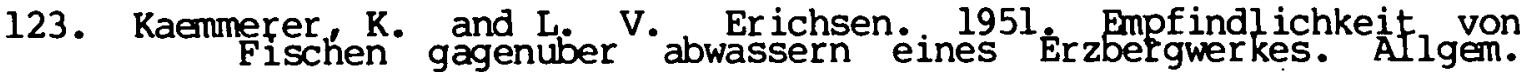
Fischerei-Zeitung $76: 1,6-7,2,32$.

124. Kaplan, H. M. and L. Yoh. 1961. Toxicity of copper for frogs. Herpetologica $17(2): 131-135$.

125. Keller, E. L., F. A. Simmonds, and P. K. Baird. 1941. Some observations on the effect of alum on certain sheet properties of paper. Paper Trade J. 112.

126. Kemp, H. T., J. P. Abrams, and R. C. Overbeck, eds. 1971. The effect of chemicals on aquatic life. Water quality criteria data book, volume 3 (draft). USEPA Water Pollut. Contr. Res. Ser. $18050 \mathrm{GWV} 5 / 71$.

127. Kemp, H. T., R. L. Little, V. L. Hulullarl, and R. L. Darby. 1973. Water quality criteria data book - vol. 5. Effects of chemicals on aquatic life. USEPA water Pollut. Contr. Res. Ser. 18050 HLA 09/73.

128. Kemp, H. T., R. G. Fuller, and R. S. Davidson. 1966. Potassium permanganate as an algiciđe. J. Amer. Water Works Assoc. $58(2): 255-263$.

129. Kihlstrom, J. E., C. Lundberg, and L. Hulth. 1971. Number of eggs and young produced by zebra fishes (Brachydanio rerio, Han.-Buch.) spawning in water containing small amounts of phenylmercur ic acetate. Env. Res. 4:355-359.

1.30. Kott. Y, and J. Edlis. 1969. Effect of halogens on algae - I. Chlorella sorokiana. Water Res. 3(4):251-256.

131. Kott, Y., G. Hershkovits, A. Shemtob, and J. B. Sless. 1966. Algicidal effect of bromine and chlorine on Chlorella pyrenoidosa. Appl. Microbiol. $14(1): 8-11$.

132. Kubena, M. 1949. The resistance of fresh-water fish toward potassium permanganate. Thesis, Coll. of Vet. Med., Brno.

133. Laroze, A. 1950. The cause of death in fish in the rearing ponds of Marao. Anais. Fac. Farm. Porto 10:5.

134. Laurent, P. J. 1956. Resistance of freshwater crustaceans to the action of sodium chromate. Verh. Intl. Ver. Limnol. 13:590-595 (1958). (in French).

135. Lawrence, J. M. 1958. Recent investigations on the use of sodium arsenite as an algicide and its effects on fish production in ponds, pp. 281-287. In: Proc. 11th Ann. Conf. Southeastern Assoc. Game Fish Commrs.

136. Learner, M. A. and R. W. Edwards. 1963. The toxicity of some substances to Nais (Oligochaeta). Proc. Soc. Water Treat. Exam. $12(3): 166-168$. 
137. Leclerc, E. 1960. The self-purification of streams and the relationship between chemical and biological tests, pp. 281-316. In: Proc. 2nd Symp. on Treatment of Waste Waters, Pergamon Press, London. (P. C. G. Issac, ed.).

138. LeCorroller, Y. 1960. Sur l'action mulluscocide due chlorure de barym sur Biomphalaria (Plenorbis) boissyi. Bull. Soc. Exot. 53:798-802.

139. Leland, H. V., E. D. Copenhaver, and L. S. Corrill. 1974. Heavy metals and other trace elements. J. Water Pollut. Contr. Fed. 46(6):1452-1476.

140. Leland, H. V., E. D. Copenhaver, and D. J. Wilkes. 1975. Heavy metals and other trace elements. J. Water Pollut. Contr. Fed. $47(6): 1635-1656$.

141. Leon, A. 1960. Carp vs. sodium dichromate. Revista Tecnica IEM (Mexico) 4, No. 29. Publ. Heal th Eng. Abst. 41:3(5), 31 (1961).

142. Lewis, S. D. and W. M. Lewis. 1971. The effect of zinc and copper on the osmolality of blood serum of the channel catfish, Ictalurus punctatus Rafinesque, and golden shiner, Notemigonus crysoleucas Mitchill. Trans. Amer. Fish. Soc. $100(4): 639-643$.

143. Liepolt, R. and E. Weber. 1958. Die Giftwirkung von Kupfersulfat auf Wasserorganismen. Wass. u. Abwass. :335-353.

144. Liu, D. H. W. and R. E. Nakatani. 1964.. Toxicity of industrial chemicals to fish, pp. 209-21l: In: Biology Research Annual Report for 1963, HW-80500. Hanford Atomic Products Operation, Richland, Wash.

145. Lloyd, R. 1960. The toxicity of zinc eulfatc to rainbow trout. Ann. Appl. Biol. 48(1):84-94.

146. Lloyd, R. and D. W. M. Herbert. 1962. The effect of the enviroment on the toxicity of poisons to fish. J. Inst. Public Health Eng. July:132-145.

147. Ludemann, D. 1953. The toxicity of manganese to fish, crabs, and animals forming the food of fish. SchrReihe Ver. Wasserhyg: No. 7 .

148. Ludwig, W. 1927. Z. Vergl. Physiol. 6.

149. Mackereth, F. J. and W. J. P. Smyly. 1951. Toxicity of copper in solution to the stone loach. Nature 158:1130.

150. Maguire, J. J., W. H. Betz, and L. D. Betz. 1956. Biological fouling in recirculating cooling systems. Ind. Eng. Chem. $48(12): 2162-2167$. 
151. Malaney, G. W., W. D. Sheets, and R. Quillin. 1959. Toxic effects of metallic ions on sewage microorganisms. Sewage Ind. Wastes $31: 1309$.

152. Maloney, T. E. and M. Palmer. 1956. Toxicity of six chemical compounds to thirty cultures of algae. Water Sewage Wks. $103(11): 509-513$.

153. Marsh, M. C. and R. K. Robinson. 1908. The treatment of fish-cultural waters for the removal of algae. Bull. Bur. Fisheries $28(2): 871-890$.

154. MAthews, A. P. 1904. The relation between solution tension, atomic volume, and the physiological action of the elements. Am. J. Physiol. 10:290-323.

155. Matthiesen, P. and A. E. Brafield. 1973. The effects of dissolved zinc on the gills of the stickleback Gasterosteus aculeatus (L.). J. Fish. Biol. 5:607-613.

156. Mcintyre, J. D. 1973. Effects of metals on fertilization and development in fish. In: Heavy metals in the environment. Seminar conducted by water Resources Research Institute, Oregon State Univ.

157. McIntyre, J. D. (unpub) . Oregon Coop. Fish. Unit, Dept. Fish. Wildlife, Oregon State Univ.

158. McIntyre, J. D. and J-M. Blanc. 19\%. Venet1c aspects of mercury toxicity for steelhead trout (Salmo gairdneri): A progress report. Proc. 52nd Ann. Conf. of the Western Assoc. State Game Fish Commrs. and Western Division, Amer. Fish. Soc.

159. McKee, J. E. and H. W. Wolt. 1963. water quality criteria, 2nd ed. Water Resources Control Bd., State of California, Publ. No. 3-A. 548 pp.

160. McKim. J. M. and D. A. Benoit. 1971. Effects of long-term exposures to copper on survival, growth and reproduction of brook trout (Salvelinus fontinalis). J. Fish. Res. Bd. Canada $28(5): \overline{655-662}$.

161. McLaughlin, R. M. 1951. New developments in bathing - water and swimming-pool sanitation. Quart. Bull. U.S. Assoc. Food and Drug Officials 15:67, Water Pollut. Absts. 25:10 (1952).

162. Meinck, F., H. Stooff, and H. Kohlschutter. 1956. Industrial waste waters (Industrie Abwasser). 2nd ed. Gustav FischerVerlg. Stuttgart 536.

163. Morgan, E. L., D. T. Burton and J. Cairns, Jr. 1971. A time until death study of bluegill sunfish (Lepomis macrochirus Raf.) exposed to simultaneous thermal and zinc stress. ABS Bull. $18(2): 47$. 
164. Morrill J B. 1963. Morphological effects of cobaltous chloride on the develophent 0 Limnaea stagnalis and Limnaea palustris. Biol. Bull. 125(3):508-522.

165. Mount, D. I. 1968. Chronic toxicity of copper to fathead minnows (Pimephales promelas, Rafinesque). Water Research $2: 215-223$.

166. Mount, D. I. and C. E. Stephen. 1969. Chronic Toxicity of copper to the fathead minnow (Pimephales promelas) in soft water. J. Fish. Res. Bd. Canada $26(9): 2449-2457$.

167. Muessig, P. H. 1974. Acute toxicity of mercuric chloride and the accumulation and distribution of mercuric chloride and methyl mercury in channel catfish (Ictalurus punctatus). M. S. Thesis, Florida State Univ. 72 pp.

168. Murdock, H. R. 1953. industrial wastes. Some data on toxicity of metals to fish life are presented. Ind. Eng. Chem. 45:99A.

169. Naumann, E. 1934. Uber die Toxizitat des Zinksulfats fur Daphnia magna. Konliga Fysiograf. Saellskapets Lund Forh. $4: 123-128$.

170. Naumann, E. 1935. The effect of some salts and mixtures of salts on Daphnia magna. Physiog. Sallsk. Lund Forh. 4:11.

171. Nebeker, A. V. and A. R. Gaufin. 1964. Bioassays to determine pesticide toxicity to the amphipod crustacean, Gammarus lacustris. Proc. Utah Acad. Sci. 41(1):64-67.

172. Nehring, D. 1962. Z. Fisch. 11:557.

173. Nehring, R. B. and J. P. Goettl, Jr 1974. Acute toxicity of a zinc-polluted stream to four species of salmonids. Bull. Envir. Contam. Toxicol. 12:464.

174. Nielson, E. and S. Wium-Andersen. 1970. Copper ions as poison in the sea and fresh water. Marine Biol. 6(2):93-97.

175. Nielson, S. S. 1939. The toxicity to fish of waste waters containing iron and copper. K. norske vidensk, Selsk. Forh. 11:233.

176. Olson, P. A. 1958. Comparative toxicity of $\mathrm{Cr}$ (VI) and $\mathrm{Cr}$ (I-II) in Salmon. Hanford Biol. Res. Ann. Rept. for 1957:215. Office of Tech. Services, U.S. Dept. of Commerce.

177. Olson, P. A. and R. F. Foster. 1956. Effect of chronic exposure to sodium dichromate on young chinook salmon and rainbow trout, pp. 35-47. In: Biology Research Annual Report for 1955, $\mathrm{HW}-41500$. Hanford Atomic Products Operation, Richland, Wash. 
178. Oshima, their ions upon young toxic action of dissolved salts and $_{\text {(Anguilla japonica). }}$ J. Imperial Fisheries Exp. Sta. (Jap.) No. 2:139-193.

179. Otto, N. E. 1970. Algaecidal evaluation and environmental study of mat-producing blue-green algae. Bur. Reclam., Office of Chief Engineer, Denver, Rept. No. REC-OCE-70-25. 31 pp.

180. Ozaki, H., K. Uematsu and K. Tanaka. 1970. Survival and growth of goldfish and carp in dilute solutions of copper sulfate. Jap. J. Ichthyol. 17(4):166-172 (Japanese, English resume); Sport Fish. Abst. 13813(1971).

181. Palmer, C. M. and T. E. Maloney. 1955. Preliminary screening for potential algicides. Ohio J. Sci. 55(1):1-8.

182. Palı ick, R. (unpub.).

103. Patrick, R., J. Cairns, Jr., and A. Scrhe1.er. 1968. The relative sensitivity of diatoms, snails, and fish to twenty common constituents of industrial wastes. Prog. Fish-Cult. $30(3): 137-140$.

184. Pfeifer, E. 1952. Magnesium narcosis in carp. Arch. Exper. Pathol. Pharm. 216:375.

185. Phillippy, C. L. 1961. Preliminary results of herbicides tested on certain aquatic plants in Florida, pp. 288-295. In: Pron. 15th Ann. Conf. Southeastern Assoc. Game Fish Commrs.

186. Pickering, Q. H. 1974. Chronic toxicity of nickel to the fathead minnow. J. Walere Pollut. Contr. Fed. 46:760.

187. Pickering, Q. H. and M. H. Gast. 1972. Acute and chronic toxicity of cadmium to the fathead minnow (Pimephales promelas). J. Fish. Res. Bd. Canada 29:1099-1106.

188. Pickering, Q. H. and $C$. Hendersun. 1964. The acute toxicity of some heavy metals to different species of warm water fishes. Proc. 19th Ind. Waste Conf. Purdue Univ., Part II. $49(2): 578-591$.

189. Pickering, Q. H. and C. Hender son. 1966. The acute toxicity of some heavy metals to different species of warm-water fishes. Air Wat. Pollut. Int. J. 10:453-463.

190. Pickering, Q. H. and W. N. Vigor. 1965. The acute toxicity of zinc to eggs and fry of the fathead minnow. Prog. Fish-Cult. $27(3): 153-157$.

191. Powers, E. B. 1917. The goldfish (Carassius carassius) as a test animal in the study of toxicity. Ill. Biol. Monogr. $4: 127-193$.

192. Powers, E. B. 1920. Influence of temperature and concentration on the toxicity of salts to fishes. Ecology 1:95-11l, 


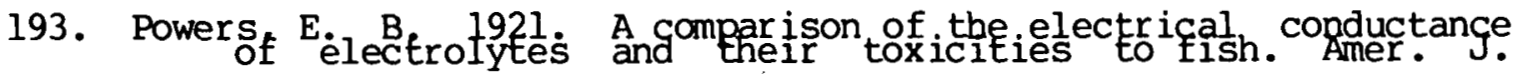
Physiol. 55:197-200.

194. Rabe, F. W. and C. W. Sappington. 1970. The acute toxicity of zinc to cutthroat trout, Salmo clarki, pp. 1-16. In: Biological Productivity of the Coeur d'Alene River as related to water quality. Completion Rept., Water Resources Res. Inst., Univ. of Idaho, Moscow; Sport Fish. Abst. 13665 (1971).

195. Rachlin, J. W. and M. Farran. 1974. Growth Response of the green alga Chlorella vulgar is to selective concentrations of zinc. Water Res. (G.B.) 8:575.

196. Rachlin, J. W. and A. Perlmutter. 1968. Response of an inbred strain of platyfish and the fathead minnow to zinc. Prog. Fish-Cult. $30(4): 203-297$.

197. Rehwolt, R., G. Bida and B. Nerrie. 1971. Acute toxicity of copper, nickel and zinc ions to some Hudson River fish species. Bull. Envir. Contam. Toxic. 6(5):445-448.

198. Roback, S. S. 1965. Environmental requirements of Trichoptera, pp. 118-126. In: Biological Problems in Water Pollution, 3rd Seminar (1962), R. A. Taft Sanit. Eng. Center, Cincinnati, Ohio, Publ. No. 999-WP-25.

199. Rudd, R. L. and R. E. Genelly. 1956. Pesticides: their use and toxicity in relation to wildlife. Calif. Dept. Fish and Game, Game Bull. No. 7.

200. Rudolfs, W., G. E. Barnes, G. P. Edwards, H. Heukelekian, E. Hurwitz, C. E. Renn, S. Steinberg, and W. F. Vaughan. Review of literature on toxic materials affecting sewage treatment processes, streams, and B.O.D. determinations. Sewage Ind. Wastes 22:1157.

201. Rudolfs, W., et al. 1953. Industrial wastes. Reinhold Publ. Co., New York.

202. Ruesink, R. G. and L. L. Smith, Jr. 1975. The relationship of the 96- hr IC50 to the lethal threshold concentration of hexavalent chromium, phenol, and sodium pentachlorophenate to fathead minnows (Pimephales promelas Rafinesque). Trans. Amer. Fish. Soc. 104:567-570.

203. Rushton, W. 1920. Fish tissues and fish poisons. Salmon and Trout Mag. No. 22:48-63.

204. Rushton, W. 1921. Biological notes. Salmon and Trout Mag. №. 25:101-117.

205. Rushton, w. 1922. Biological notes. Salmon and Trout Mag. No. $28: 42-51$.

206. Rushton, W. 1949. Biological notes. Salmon and Trout Mag. $125: 57-61$. 
207. Sanborn, N. H. 1945. The lethal effect of certain chemicals on fresh water fish. Canning Trade 67:49,10-12,26.

208. Sanders, H. O. and O. B. Cope. 1968. The relative toxicities of several pesticides to naiads of three species of stoneflies. Limnol. Oceanogr. 13(1):112-117.

209. Sangalang, G. B. and M. J. O'Halloran. 1972. Cadmium-induced testicular injury and alterations of androgen synthesis in brook trout. Nature 240:470-471.

210. Sappington, C. W. 1969. The acute toxicity of zinc to cutthroat trout. M.S. Thesis, Dept. of Biol., University of Idaho.

211. Schaperclaus, W. 1941. Fischktankheiten. Gustav Wenzel \& Sohn, Braumschweig, 261 pp.

212. Schaut, G. G. 1939. Fish catastrophes during drought. J. A.W.W.A. $31: 771$.

213. Schiffman, R. H. and P. O. Fromm. 1959. Chromium-induced changes in the blood of rainbow trout, Salmo gairdnerii. Sewage Ind. Wastes 31:205.

214. Schroeder, H. A. 1965. The biological trace elements or peripatetics through the periodic table. J. Chron. Dis. 18:217-228.

215. Schweiger, G. 1957. Dle tuxiculüische Einwirkung von Schwermetallsalzen auf Fische und Fischnahrtiere. Arch. Fischereiwissenschaft $8: 54-78$.

216. Shaw, T. L. and V. M. Brown. 1974. The toxicity of some forms of copper to rainbow trout. Water Research 8:377-382.

217. Shaw, W. H. R. and B. Grushkin. 1967. The toxicity of metal ions to aquatic organisms. Arch. Biochem. Biophys. $67(2): 447-452$.

218. Shaw, W. H. R. arid B. R. Lowrance. 1956. Bioassay for the estimation of metal ions. Anal. Chem. 28:1164.

219. Sheets, W. D. 1957. Toxicity studies of metal-finishing wastes. Sewage Ind. Wastes 29:1380.

220. Simonin, P. and A. Pierron. 1937. Acute toxicity of fluorine compounds. Compt. Rend. Soc. Biol. 124:133.

221. Skidmore, J. F. 1965. Resistance to zinc sulfate of the zebrafish (Brachydanio rerio Hamilton-Buchanan) at different phases of its life history. Ann. Appl. Biol. 56(1):47-53.

222. Skidmore, J. F. 1966. Resistance to zinc sulfate of zebrafish (Brachydanio rerio) embryos after removal or rupture of the outer egg membrane. J. Fish. Res. Bd. Canada $23(7)$ : 1037-1041. 
223. Skidmore, J. F. 1967. Oxygen uptake by zebrafish (Brachydanio rerio) at different ages in relation to zinc sulfate resistance. J. Fish. Res. Bd. Canada $24(6): 1253-1267$.

224. Skidmore, J. F. and P. W. A. Tovell. 1972. Toxic effects of zinc sulfate on the gills of rainbow trout. Water Res. $6(3): 217-230$.

225. Slonim, C. B. and A. R. Slonim. 1973. Effect of water hardness on the tolerance of the guppy to beryllium sulfate. Bull. Env. Contam. Toxicol. 10:295-301.

226. Smith, M. W. 1939. Copper sulfate and rotenone as fish poisons. Trans. Amer. Fish. Soc. 69:141-157.

227. Smith, J. D., et al. 1973. Mercury in sediments from the Thames estuary. Environ. Poll. 4:2,153.

228. Somers, E. 1961. The fungitoxicity of metal ions. Ann. Appl. Biol. $49(2): 246-253$.

229. Southgate, B. A. 1948. Treatment and disposal of industrial waste waters. Dept. of Sci. Ind. Res., H. M. Stationery Office, London.

230. Sparks, R. E., J. Cairns, Jr., and A. G. Heath. 1972. The use of bluegill breathing rates of detect zinc. Water Res. $6: 895-911$.

231. Speyer, M. R. and G. Leduc. 1975. Effects of arsenic trioxide on growth of rainbow trout. Presented at the Internat. Conf. on Heavy Metals in the Envir., Toronto.

232. Sprague, J. B. 1964. Lethal concentrations of copper and zinc for young Atlantic salmon. J. Fish. Res. Bd. Canada $21(1): 17-26$.

233. Sprague, J. B. 1964. Avoidance of copper-zinc solutions by young salmon in the laboratory. J. Water Pollut. Control Fed. $36(8): 990-1004$.

234. Sprague, J. B. 1968. Promising anti-pollutant: chelating agent NTA protects fish from copper and zinc. Nature $220(5174): 1345-1346$.

235. Sprague, J. B. 1968. Avoidance reactions of rainbow trout to zinc sulphate solutions. Water Res. (2):236-372.

236. Sprague, J. B. and B. A. Ramsay. 1965. Lethal levels of mixed copper-zinc solutions for juvenile salmon. J.' Fish. Res. Bd. Canada $22(2): 425-432$.

237. Springer, P. F. 1957. Effects of herbicides and fungicides on wildlife. N. Car. Pest. Man., N. Car. St. Coll., Ralej.gh. 87 pp. 
238. Sreenivasan, A. and R. S. Raj. 1963. Toxicity of zinc to fish. Current Sci. (India) 32(8):363; Chem. Abst. $59: 14338 b(1963)$.

239. Stanley, R. A. 1974. Toxicity of heavy metals and salts to Eurasian watermilfoil (Myriophyllum spicatum L.). Arch. Environ. Contam. Toxicol. 2:331-341.

240. State of Washington. 1944. 64th Ann. Rep. Fish.

241. Surber, E. W. 1932. Controlling vegetation in fish ponds with sodium arsenite. Bur. of Fisheries Investigatonal Rept. 11 .

242. Surber, E. W. 1943. Weed control in hard-water ponds with copper oulfatc and sodium arsenite, Trans. N. Aller. Wildl. Conf. 8:132-141.

243. Surber, E. W. 1949. Control of aquatic plants in ponds and lakes. U.S. Fish Wildl. Serv., Fish. Leaflet No. 344. $26 \mathrm{pp}$.

244. Surber, E. W. 1965. Water quality criteria for freshwater fishes, pp. 435-436. In: Proc. 16th Ann. Conf. Southeastern Assoc. Game Fish Commr. (1965). (Abstract).

245. Surber, E. W. and M. H. Everhart. 1950. Biological effects of nigrosine used for control of weeds in hatchery ponds. Progr. Fish. Cult 12:135.

246. Surber, E. W. and O. L. Meehan. 1931. Lethal concentrations of arsenic for certain aquatic organiems. Trans. Amer. Fish. Soc. 61:225.

247. Sutton, D. L. and R. D. Blackburn. 1971. Uptake of copper by parrotfeather. Weed. Sci. 19:282.

248. larzwell, C. M. and C. Henderson. 1956. The toxieity of some of the less common metals to fishes. Trans., Seminar on Sanitary Engineering Aspects of the Atomic Energy Industry, Robt. A. Taft San. Engrg. Center. TID-7517.

249. Tarzwell, C. M. and C. Henderson. 1960. Toxicity of less common metals to fishes. Industrial Wastes 5:12.

250. Thomas, A. 1915. Effects of certain metallic salts upon fishes. Trans. Amer. Fish. Soc. 44:120-124.

251. Trama, F. B. 1954. The acute toxicity of copper to the common bluegill (Lepomis macrochirus Rafinesque). Notulae Natur. $257: 1-13$.

252. Trama, F. B. and R. J. Benoit. 1960. Toxicity of hexavalent chromium to bluegills. J. Water Pollut. Control Fed. $32(8): 868-877$. 
253. Turnbull, H., J. G. DeMann, and R. F. Weston. 1954. Toxicity of various refinery materials to fresh water fish. Symp. on Waste Disposal in the Petrol. Ind., Ind. Eng. Chem. $46: 324$.

254. Turnbull-Kemp, P. St. J. 1958. Trout in southern Rhodesia. V. On the Toxicity of copper sulfate to trout. Rhodesia Agr. J. $55(6): 637-640$.

255. U.S.E.P.A 1972. Quarterly Res. Rept., March 31, 1972. National Wat. Qual. Lab., Duluth, Minn.

256. U.S.E.P.A. 1972. Quarterly Res. Rept.,. June 30, 1972. National Wat. Qual. Lab., Duluth, Minn.

257. Uspenskaya, V. I. 1946. Experimental observations on the influence of mercury compounds on aquatic organisms. Hyg. and Sanit. (U.S.S.R) $11: 11,1-5$.

258. Velsen, F. J. P. and D. F. Alderdice. 1967. Toxicities of two insecticides to young coho salmon. J. Fish. Res. Bd. Canada 24:1173-1175.

259. Vivier, P. and M. Nisbet. 1965. Toxicity of some herbicides, insecticides, and industrial wastes, pp. 167-169. In: Biological Problems in Water Pollution, 3rd Seminar (1962), R. A. Taft Sanit. Eng. Center, Cincinnati, Ohio; USPHS Publ. No. 999-WP-25.

260. Vladimirov, V. I. 1969. Dependence of the embryonic development. and viability of the carp on the trace element zinc. Problems in Ichthyology 9(5):681-696. (English Translation).

261. Waitz, S. and J. B. Lackey. 1959. morphological and biochemical studies on the organism Sphaerotilus natans. Eng. Progr. Univ. Fla. 13:10, Leaflet No. 114; Quart. J. Fla. Acad. Sci. 21:335.

262. Wallen I. E., W. C. Greer, and R. Lasater. 1957. Toxicity to Gambusia affinis of certain pure chemicals in turbid waters. Sewage Ind. Wastes 29:695.

263. Waller, W. T. and J. Cairns, Jr. 1972. The use of fish movement palteris to monitor zinc in water. Water Res. $6: 257-269$.

264. Warnick, S. L. and H. L. Bell. 1969. The acute toxicity of some heavy metals to different species of aquatic insects. J. Water Pollut. Contr. Fed. 4l:280-284.

265. Warrick, L. F., H. E. Wirth, and W. Van Horn. 1943. Control of microorganisms and aquatic vegetation. Water Works Sewage $90(7)$ : 267-272; B10l. Abst. I8: $1330(1944)$. 


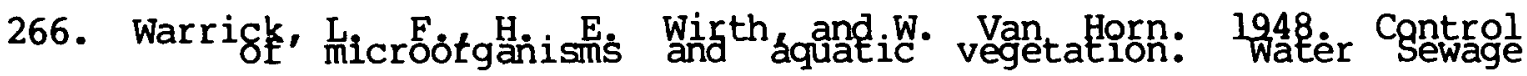
Works. $95(4)$ : R147-150.

267. Water Pollut. Res., 1959. 1960. H.M.S.O., London.

268. Water quality criteria. 1968. FWPCA, U.S. Dept. of Interior, Washinton, D.C.

269. Water quality criteria data book. 1971. Vol. 2. USEPA. 18010 DPV $07 / 71$.

270. Weir, P. A. and C. H. Hine. 1970. Effects of various metals on behavior of conditioned goldfish. Arch. Environ. Health $20: 45-51$.

271. Western Fish Toxicology Station. (Unpub.). National Envir. Res. Center, Corvallis, Oregon.

272. Whitley, L. S. 1968. The resistance of tubificid worms to three common pollutants. Hydrobiologia $32(1-2): 193-205$.

273. Whitton, B. A. 1970. Toxicity of zinc, copper and lead to Chlorophyta from flowing waters. Arch. Mikrobiol. $72(4): 353-360$.

274. Wiebe, A. H., J. G. Burr, and H. E. Faubion. 1934. The problem of stream pollution in Texas with special reference to salt waters from oil fields. Trans. Amer. Fish. Soc. $64: 81$.

275. Willford, W. A. 1966. Toxicity of 22 therapeutic compounds to six fishes. In: Investigations in Fish Control, No. 18. U.S. Flsh. Wildl. Serv., Bur. Gport Fish. Widl., Rerour. Pinhl. 35. $10 \mathrm{pp}$.

276. Williams, L. G. and D. I. Mount. 1965. Influence of zinc on periphyton communities. Amer. J. Bot. $52(1): 25-34$.

277. Wilson, R. C. H. 1972. Prediction of copper toxicity in receiving waters. J. Fish. Res. Bd. Canada $29(10): 1500-1502$.

278. Woelke, C. E. 1961. Bloastay - Lhe bivalve lorvor tool. Proc. 10th Pacific Northwest Symp. on Water Pollut. Research. Toxicity in the aquatic environment. Portland, Oregon.

279. Wurtz, A. 1945. The action of boric acid on certain fish: trout, roach, rudd. Ann. Sta. Bent. Hydrobiul. Appl. 1, 179; Water Pollut. Res. 20:1653(1947).

280. Wurtz, C. B. 1962. Zinc effects on freshwater mollusks. Nautilus $76(2): 53-61$. 
281. Wurtz, C. B. and C. H. Bridges. 1961. Preliminary results from macroinvertebrate bioassays. Proc. Pa. Acad. Sci. $35: 51-56$.

282. Young, R. T. 1923. Resistance of fish to salts and alkalinity. Amer. J. Physiol. 63:373.

283. Young, R. G. and D. J. Lisk. 1972. Effect of copper and silver ions on algae. J. Water Pollut. Control Fed. $44(8)$ : $1643-1647$.

284. Zitko, V. 1975. Toxicity and pollution potential of thallium. Sci. Total Environ. 4:185-192.

285. Zitko, V., W. V. Carson, and W. G. Carson. 1973. Prediction of incipient lethal levels of copper to juvenile Atlantic salmon in the presence of humic acid by cupric electrode. Bull. Env. Contam. Toxicol. 10:265-271.

286. Zitko, V., W. V. Carson, and W. G. Carson. 1975. Thallium: occurrence in the environment and toxicity to fish. Bull. Env. Contam. Toxicol. 13:23-30.

287. Herbert, D. W. M. and D. S. Shurben. 1964. The toxicity to fish of mixtures of poisons. I. Salts of ammonia and zinc. Ann. Appl. Biol. 53:33-41.

288. Herbert, D. W. M. and A. C. Wakeford. 1964. The susceptibility of salmonid fish to poisons under estuarine conditions-I. Zinc sulfate. Intl. J. Air Water Pollut. $8: 251-256$.

289. Herbert, D. W. M., D. H. M. Jordon and R. Lloyd. 1965. A study of some fishless rivers in the industrial midlands. J. Proc. Inst. Sewage Purif., London 6:569-582.

290. Hermann, E. R. 1959. Toxicity index for industrial wastes. Ind. Eng. Chem. 51:84A.

291. Hervey, R. J. 1949. Effect of chromium on the growth of unicellular chlorophycease and diatoms. Bot. Baz. $111(1): 1-11$.

292. Heukelekian, H. and I. Gellman. 1955. Studies of biochemical oxidation by direct methods. IV. Effect of toxic metal ions on oxidation. Sewage Ind. Wastes 27:70.

293. Hiltibran, R. C. 1967. Effects of some herbicides on fertilized fish eggs and fry. Trans. Amer. Fish. Soc. 96:414-416.

294. Hodgson, E. S. 1951. Reaction thresholds of an aquatic beetle, Laccophilus maculosis, to salts and alcohols. Physiol. 2001. 24:131. 
295. Holland, G. A., J. E. Lasater, E. D. Newmann, and W. E. Eldridge. 1960. Toxic effects of organic pollutants on young salmon and trout. State of Washington, Dept. of Fisheries, Res. Bull. No. 5. 264 pp.

296. Hubschman, J. H. 1967. Effects of copper on the crayfish Orconectes rusticus (Girard). I. Acute toxicity. Crustaceana 12:33-42.

297. Ingols, R. S. 1954. Toxicity of mercuric chloride, chromic sulfate, and sodium chromate in the dilution BOD test. Sewage Ind. Wastes $26(4): 536-538$.

298. Cairns, J., Jr. 1957. Environment and time in flsh toxicity. Ind. Wastes 2:1-4.

299. Cairns, J., Jr. 1965. Biological concepts and industrial waste disposal problems. Proc. 20th Ind. Waste Conf., Purdue Univ. $49(4): 49-59$.

300. Sprague, J. B. 1965. Effects of sublethal concentrations of zinc and copper on migration of Atlantic salmon, pp. 332-333. In: Biological Problems in Water Pollution, 3rd Seminar (1962), R. A. Taft Sanitary Eng. Center, Cincinnati. USPHS Publ. No. 999-WP-25.

301. Shaw, T, L. and V. M. Brown. 1971. Heavy metals and the fertilization of rainbow trout eggs. Nature $230(5291): 251$.

302. Jones, J. R. E. 1937. J. Exp. Biol. $14(3)$.

303. Jones, J. R. E. 1947. The reactions of Pyyusteus pungitiug L. tu Luxic solutiona: J. Exp. Biol. 25:22-34.

304. Fitzgerald, G. P., G. C. Giluff, and F. Skoog. 1952. Studies on chemicals with selective toxicity to blue-green algae. Sewage Ind. Wastes $24(7): 888-897$.

305. Whitworth, W. R. and T. H. Lane. 1969. Effects of toxicants on community metaboilsin in puols. Limnol. Occanogr. $14(1): 53-58$.

306. Mintyre, J. D. 1972. Toxicity of methylmercury for steplhead trout sperm. Bull. Envir. Contam. Toxicol.

307. Thomas, A. 1924. Studies on the absorption of metallic salts by fish in their natural habitat. II. The absorption of nickel by Fundulus heteroclitus. J. Biol. Chem. 58,671-674.

308. Skidmore, J. F. 1964. Toxicity of zinc compounds to aquatic animals, with special reference to fish. Quart. Rev. Biol. $39: 227-248$. 
309. Mount, D. I. 1966. The effect of total hardness and pH on the acute toxicity of zinc to fish. Int. J. Air Wat. pollut. $10: 49-56$.

310. Cairns, J., Jr. and A. Scheier. 1957. The effects of temperature and hardness of water upon the toxicity of zinc to the common bluegill (Lepomis macrochirus Raf.). Notulae Naturae Acad. Nat. Sci. Phila., 299. 12 pp.

311. Pickering, Q. H. 1968. Some effects of dissolved oxygen concentrations upon the toxicity of zinc to the bluegill, Lepomis macrochirus Raf. Water Res. $2(3): 195-213$.

312. Skidmore, J. F. 1970. Respiraton and osmoregulation in rainbow trout with gills damaged by zinc sulphate. J. Exp. Biol. $52: 481-494$.

313. Sparks, R. E., J. Cairns, Jr., R. A. McNabb, and G. Suter, II. 1972. Monitoring zinc concentrations in water using the respiratory response of bluegills (Lepomis macrochirus Rafinesque). Hydrobiologia $40(3): 361-369$. 
Table 8. Program and output listing to subset data base for only those records with an organism name within crustaceans.

A. Program listing

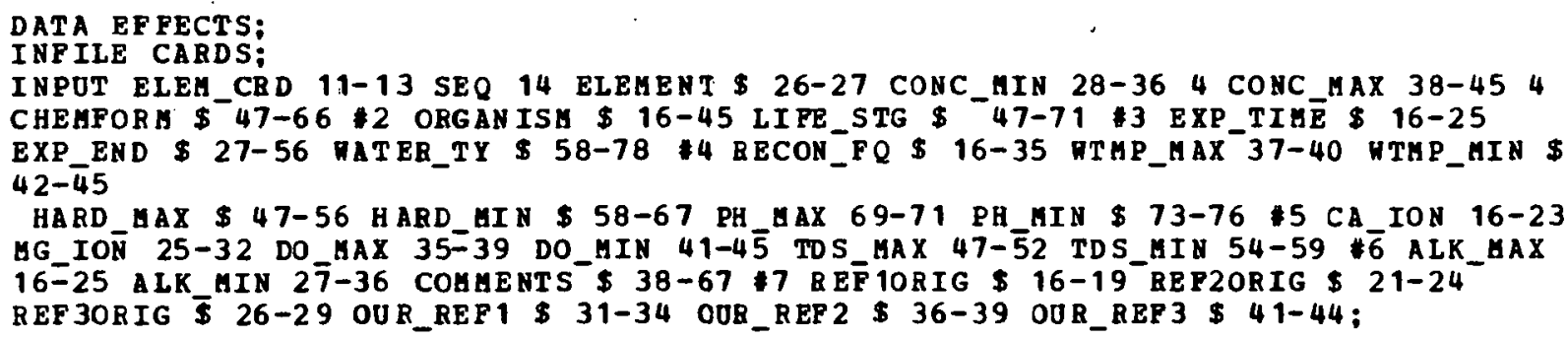

TITLE TOXIC EFECTS KOK CEOSTACEANS: PROC PRINT DATA=EPFECTS: 
Table 8 (cont'd). B. Portion of output from previous program.

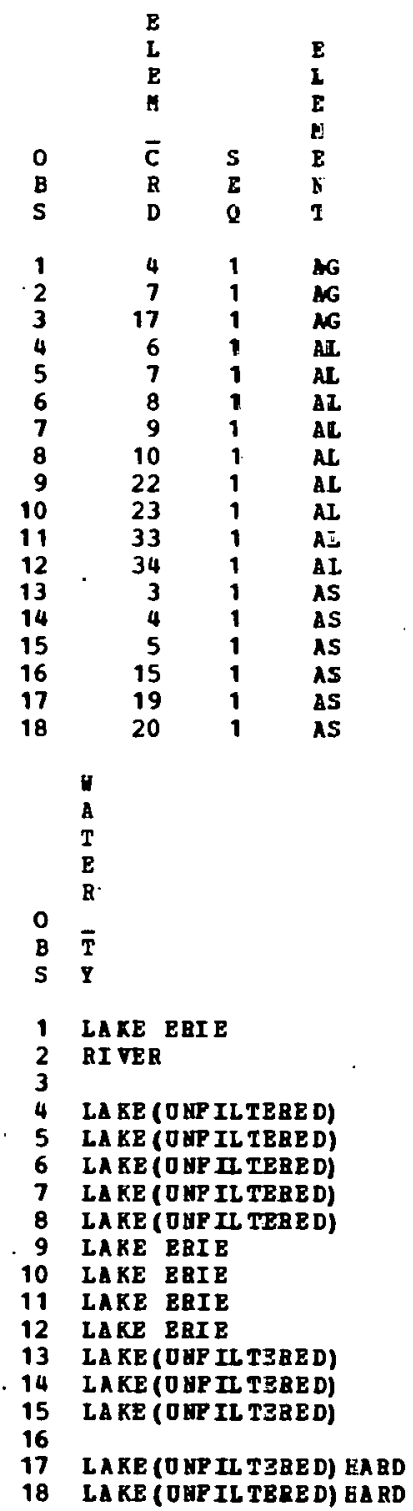

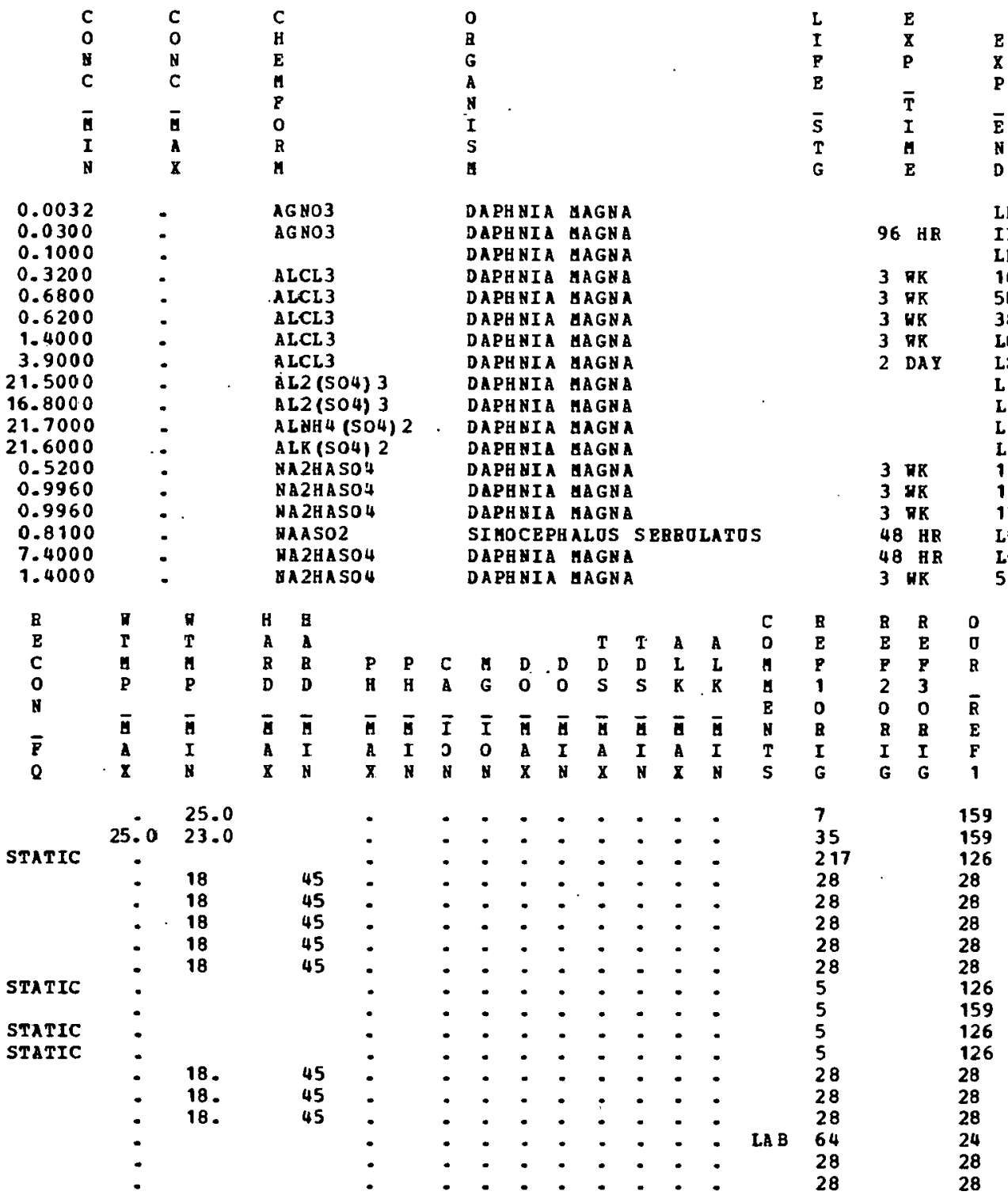


THIS PAGE

WAS INTENTIONALLY

LEFT BLANK 
ORNL/TM-5793

\section{INTERNAL DISTRIBUTION}

1. S. M. Adams

2. R. M. Anderson

3-22. S. I. Auerbach

23. L. W. Barnthouse

24. B. G. Blaylock

25. R. W. Brocksen

26. R. L. Burgess

27-29. J. A. Carter

30-35. C. C. Coutant

36. F. L. Culler

37-46. R. M. Cushman

47. B. J. Dozier

48: G. K. Eddlemon

49-58. ' C. W. Gehrs

59. J. M. Giddings

60. S. B. Gough

61. S. E. Herbes

62-71. S. G. Hildebrand

72. D. D. Huff

73. J. R. Hyndman

74. J. A. Klein

75. R. K. Kroodsma
76. J. M. Loar

77. R. J. 01son

78. B. R. Parkhurst

79. D. C. Parzyck

80. H. Postma

81. D. E. Reichle

82. N. Revis

83. C. R. Richmond

84. R. D. Roop

85. F. S. Sanders

86. G. R. Southworth

87-106. R. H. Strand

107. E. G. Struxness

108. A. T. Szluha

109. R. I. Van Hook

110. Biology Library

111. ORNL Patent Office

112-113. Central Research Library

114. Lab Records, ORNL-RC

115-116. Lab Records Dept.

117. ORNL Y-12 Technical

Library

\section{EXTERNAL DISTRIBUTION}

118. Research and Technical Support Division, ERDA-ORO.

119. T. M. Albert, Office of Environmental Information Systems, Energy Research and Development Admin istration, Washington, DC 20545.

120. D. S. Ballantine, Division of Biomedical and Environmental Research, Energy Research and Development Administration, Washington, DC 20545.

121. N. F. Barr, Division of Terhnology Overview, Energy Research and Development Administration, Washington, DC 20545.

122. K. Biesinger, Environmental Protection Agency, 6201 Congdon Boulevard, Duluth, MN 55804.

123. C. E. Carter, division of Biomedical and Environmental Research and Development Administration, Washington, DC 20545.

124. D. Coffin, Environmental Protection Agency, Research Triangle Park, NC 27711.

125. C. Comar, EPRI, P. 0. Box 10412, Palo Alto, CA 94304.

126. C. W. Edington, Division of Biomedical and Environmental Research, Energy Research and Development Administration, Washington, DC 20545. 
127. R. Franklin, Division of Biomedical and Environmental Research, Energy Research and Development Administration, Washington, DC 20545.

128. C. W. Hall, Environmental Protection Agency, 401 M Street S.W., Washington, DC 20460.

129. H. Hamilton, Division of Biomedical and Environmental Research, Energy Research and Development Administration, Washington, DC 20545 .

130. D. C. Hutchinson, Union Carbide Corporation, Saw Mill River. Road 100C, Tarrytown, NY 10591.

131. R. M. Jimeson, Division of Technology Overview, Energy Research and Development Administration, Washington, DC 20545.

132. H. Jones, Major Facility Project Management, Energy Research and Development Administration, Washington, DC 20545.

1.33. Paul Karmowski, Director, Institute of Ecological Studies, University of North Dakota, Grand Forks, ND 58201.

134. R. D. Kerr, Laramie Energy Research Center, P. 0. Box 3395, University Station, Laramie, WY 82071.

135. J. L. Liverman, Division of. Biomedical and Environmental Research, Energy Research and Development Administration, Washington, DC 20545.

136. S. E. Manahan, Department of Chemistry, University of Missouri, Columbia, MO 65201.

137. S. Marks, Battelle Pac if ic Northwest Laboratories, P. 0. Box 999, Richland, WA 99352.

138. M. J. Massey, Carnegie-Mellon University, Schen ley Park, Pittsburgh, PA 15213.

139. W. E. Mott, Division of Biomedical and Environmental Research, Energy Research and Development Administration, Washington, DC 20545.

140. D. I. Mount, Environmental Protection Agency, 6201 Congdon Boulevard, Duluth, MN 55804.

141. Váun A. Newi11, M.D., Exxon Corporation, P. 0. Box 45, Linden, NJ 07036 .

142. W. S. Osburn, Uivis1or ur Biomedical and Envirnnmental Research, Energy Research and Development Administration, Washington, DC 20545 .

143. R. W. Perkins, Battelle Pacific Northwest Laboratories, P. 0. Box 999, Richland, WA 99352.

144. G. J. Rausa, Envirumimental Protection Agency, 401 M Street S.W., Washington, DC 20460.

145. M. J. Rei11y, Division of Environmental and Socioeconomic Progranis, Energy Research and Development Administration, Washington, DC 20545.

146. W. Reiners, National Science Foundation, $1800 \mathrm{G}$ Street, N.W., Washington, DC 20550.

147. J. J. Reisa, Council on Environmental Quality, 722 Jackson Place, Washington, DC 20006. 
148. G. W. Saunders, Jr., Division of Biomedical and Environmental Research, Energy Research and Development Administration, Washington, $D C 20545$.

149. J. J. Schmidt-Collerus, Denver Research Institute, University of Denver, Denver, CO 80210.

150. R. L. Scott, Pittsburgh Energy Research Center, 4800 Forbes Avenue, Pittsburgh, PA 15213.

151. J. A. Sherk, U.S. Department of the Interior, Fish and Wildlife Service, Office of Biological Services, Washington, DC 10240.

152. N. Shimp, Illinois State Geological Survey, Natural Resources Building, Sixth and Peabody, Urbana, IL 61801.

153. M. Singer, Division of Environmental and Socioeconomic Programs, Energy Research and Development Administration, Washington, DC 20545.

154. D. H. Siade, Division of Biomedical and Environmental Research, Energy Research and Development Administration, Washington, DC 20545.

155. J. Swinebroad, Division of Biomedical and Environmental Research, Energy Research and Development Administration, Washington, DC 20545.

156. R. G. Thomas, Los Alamos Scientific Laboratory, P. O. Box 1663, Los Alamos, NM 87545.

157. D. J. Viviani, Environmental Protection Agency; $401 \mathrm{M}$ Street S.W., Washington, DC 20460.

158. H. R. Wasson, Division of Technology Overview, Energy Research and Development Administration, Washington, DC 20545.

159. R. L. Watters, Division of Biomedical and Environmental Research, Energy Research and Development Administration, Washington, DC 20545.

160. J. H. Wilson, Office of Environmental Information Systems, Energy Research and Development Administration, Washington, DC 20545.

161. R. W. Wood, Division of Biomedical and Environmental Research, Energy Research and Development Administration, Washington. DC. 20545.

162-188. Technical Information Center, Oak Ridge, TN 37830. 
THE TOXICITY OF 35 TRACE ELEMENTS IN COAL TO FRESHWATER BIOTA: A DATA BASE WITH AUTOMATED RETRIEVAL CAPABILITIES

R. M. Cushman, S. G. Hildebrand,

R. H. Strand, and R. M. Anderson

MICROFICHE ENCLOSED 